\title{
The Composition of Oxygen Functionals Groups at the Surface of Carbon-Based Graphitic Anode
}

\author{
Nadia N. Intan† and Jim Pfaendtner*†t \\ $\dagger$ Department of Chemical Engineering, University of Washington, Seattle, Washington 98195 \\ + Physical Sciences Division, Pacific Northwest National Laboratory, Richland, Washington 99352 \\ *Email: jpfaendt@uw.edu
}

\begin{abstract}
In lithium-ion batteries (LIBs), the quality of a solid electrolyte interphase (SEI) that forms at the electrode/electrolyte interface substantially affects the stability and lifetime of the devices. One of the major determinants of the morphology and properties of SEI is the surface structure and composition of the graphitic anode used. The presence of oxygenated surface groups at the graphitic anode facilitates the formation of SEI at the interface that stabilizes LIBs. A series of DFT calculations reveal that at typical operating conditions (temperature, $\mathrm{pH}$ ) of LIBs, the $(11 \overline{2} 0)$ edge facet of graphite anode will be fully oxygenated, while the basal sites remain unsaturated. The oxygen functional groups at the edge sites are comprised of mostly hydroxyl and ketonic groups, with carboxyl and carbonyl groups are present in small amounts. Furthermore, we observe transformation of carbonyl group into ketonic group in the presence of empty surface carbon sites, which further stabilize the graphite surface. Meanwhile, carboxyl groups are more stable when all surface sites within a carboxyl layer are all populated. On the contrary to the edge plane, a small amount of oxygen functional groups may be forced to adsorb on the basal surface upon application of an external potential.
\end{abstract}

Keywords: DFT, Pourbaix diagram, graphite anode, LIBs 


\section{Introduction}

Graphite is a widely used material across a spectrum of applications, ranging from aircraft engines, coatings, carbon filters to electrochemical and energy storage systems. [1-3] Graphite is extensively used as the main component of the anode in lithium ion batteries (LIBs) due to its supreme stability and low cost. [4] Graphite has a hexagonal structure that belongs to the $P 6_{3} / m m c$ space group with cell parameters of a $=$ $\mathrm{b}=2.464 \AA$ and $\mathrm{c}=6.771 \AA$, with interlayer spacing of $3.354 \AA$. Graphite layers are stacked in an $\mathrm{AB}$ sequence, with the atoms of the $B$-layer are shifted by $1 / 3$ of the lattice vectors a and $b$ from the atoms of the A layers, and are mainly held together by London dispersion forces. The three-dimensional nature of graphite ensures the existence of multiple surfaces which determine its macroscopic crystal shape. However, the hexagonal symmetry of a graphite unit cell reduces the number of low-index surfaces to only

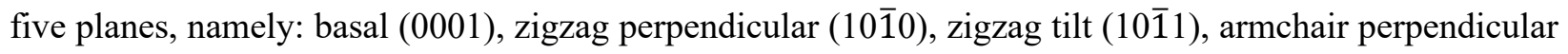
$(11 \overline{2} 0)$ and armchair tilt $(11 \overline{2} 1)$ facets. [5] Both basal and edge facets of graphite have been studied extensively. In terms of surface energy, the armchair terminations of edge graphite are $\sim 1.6 \mathrm{~J} / \mathrm{m}^{2}$ more stable than the zigzag terminations. Meanwhile, the surface energy of (0001) basal graphite is only 0.18 $\mathrm{J} / \mathrm{m}^{2}$, which is the lowest of all terminations.

However, for graphite to be suitable for usage as anode in LIBs, it needs to undergo several processes to improve its properties, such as surface functionalization and lithium intercalation. Surface functionalization helps prevent graphite exfoliation, thereby bringing further stabilization to the anode. [6] Graphite surface functionalization changes the hybridization of the surface carbon atoms from planar $\mathrm{sp}^{2}$ to tetrahedral $\mathrm{sp}^{3}$ which makes a new $\mathrm{p}$ atomic orbital available to allow binding with functional groups. [7] One of the most common functional groups that are used in graphite are those containing oxygen functional groups such as hydroxyl $(-\mathrm{C}-\mathrm{OH})$, ketonic $(-\mathrm{C}=\mathrm{O})$, carboxyl $(-\mathrm{C}(-\mathrm{OH})=\mathrm{O})$, and carbonyl $(-\mathrm{C}(-$ $\mathrm{H})=\mathrm{O}$ ) groups. [8-10] Graphite surface functionalization with oxygen functional groups increases the dspacing of graphite, which enables faster lithium intercalation [11] and therefore, hastens charging. The presence of surface oxygen functional groups also stabilizes the electronic delocalization in the anode 
$[12,13]$ by lowering the HOMO-LUMO bandgap between the graphite anode and solvent molecules. The lowering of the HOMO-LUMO bandgap is important in promoting the binding of electrolyte molecules onto the surface, which facilitates the formation of the solid electrolyte interphase (SEI) at the electrode/electrolyte interface that stabilizes LIBs. [14] Previous experimental work has also shown that the incorporation of oxygen functional groups significantly improves the energy density of the anode electrodes. [15] Additionally, controlled oxidation of graphitic surfaces allows the fine tuning of the electronic, optical, and mechanical properties of the anode. [16]

Different carbon-based materials (e.g.: graphite, graphene, nanotube, fullerene) have been found to incorporate different oxygen functional groups at different sites and at varying extents of oxidation when these oxygen-functionalized surfaces are synthesized. [17-19] For example, in the case of a graphene oxide, epoxide and hydroxyl functional groups are present in large amounts on the basal side of graphene oxide, while small amounts of carbonyl and carboxyl groups are distributed at the edges of the layer. [20] Upon mild thermal treatment, the oxygen functional groups may migrate towards the edge of the graphene layer. [15] Although there have been many studies on the structures of graphite under various experimental conditions, the structure and composition of graphite on an anode surface remains elusive. It is difficult to experimentally determine the exact anode surface structure and composition because of the easy buildup of the SEI that covers the anode surface after a few charge/discharge cycles of LIBs. [12] Despite this difficulty, a complete understanding of the interfacial structure, including the composition of surface functionalization, is of paramount importance in improving the performance of LIBs, as it plays a crucial role in the lithium intercalation rate, the charge/discharge performance, and dendrite formation. [21] Furthermore, it also has a significant impact on the structures, morphologies and properties of the SEI, $[22,23]$ which in turn affects the quality and lifetime of the LIBs.

To address these knowledge gaps, we computationally investigated the composition of oxygen functional groups on graphite surfaces, such as those that can be found in the anode of LIBs. We use the computational hydrogen electrode (CHE) [24] method to construct the surface Pourbaix diagram [25] to 
assess the stability of various oxygen functional groups at both edge and basal sites of a graphite surface. We vary the surface coverage of oxygen functional groups on graphite surfaces [26] to quantify the propensity of a graphite surface to be covered by a specific oxygen group. From this, new insights were obtained that help explain the competition between different oxygen functional groups for adsorption sites at the graphite surface in LIBs. Calculations of the extent of surface coverage for each oxygen functional group on graphite surfaces reveal not only the composition of the graphite surface, but also provide new fundamental knowledge on the interfacial chemical reactions. The remainder of this paper is organized as follows: First, we provide the model systems used in our calculations and describe the procedure used to construct the surface Pourbaix diagram. We then present our results and discuss on our findings with a perspective on how this information may possibly guide the material design of a graphite anode to obtain greater control of the performance of LIBs. Finally, we discuss how this knowledge could be extended to other electrochemical systems that also utilize graphite for one of their components, such as sodium-ion batteries (NIBs), lithium-ion hybrid supercapacitors (LICs), and fuel cells.

\section{Computational Methods}

All calculations were carried out within the plane-wave density-functional-theory (DFT) framework as implemented in the NWChem code. [27] The exchange and correlation energies were calculated using the Perdew-Burke-Ernzerhof (PBE) functional within the generalized gradient approximation. [28] The PBE functional was corrected for dispersion interactions using the Grimme approach (PBE-D3 with BJ damping). [29] Hamann pseudopotentials were used for oxygen, carbon and hydrogen. [30,31] The Hamiltonian matrix was diagonalized through the Monkhorst Pack scheme, using a $2 \times 2 \times 1$ k-mesh. [32]

The kinetic cutoff energies of 60 and 120 Ry were applied to expand the Kohn-Sham electronic wave functions and charge density, respectively. Both adsorbates and the top two layers of the graphite surface were allowed to fully relax during structure optimizations, while the carbon atoms in the third graphite layer were fixed in the bulk position. 
The graphite surfaces were modelled as periodic slabs comprised of two and five atomic layers for (0001) basal plane and $(11 \overline{2} 0)$ armchair edge, respectively. Both (0001) and (11 $\overline{2} 0)$ facets are the low-index graphite surfaces with the lowest surface energies for basal and edge surfaces, respectively. [5] Furthermore, in addition to the lowest surface energy argument, the (1120) armchair facet was chosen to represent the edge surface as the armchair facets are found to be more suitable for LIB applications than the zigzag facets, which promotes lithium dendrite formation and have detrimental impact on charge/discharge rates. [21] The graphite surfaces were modeled in a box of 13.54 x $12.70 \AA^{2}$ for (112̄0) edge and $9.78 \times 12.70 \AA^{2}$ for basal plane with a vacuum gap of at least $10 \AA$ in the $z$-direction. [33] Surfaces were functionalized with $(\mathrm{C}-\mathrm{OH} / \mathrm{C}-\mathrm{H}),(\mathrm{C}=\mathrm{O} / 2 \mathrm{C}-\mathrm{H}),\left(\mathrm{O}=\mathrm{C}-\mathrm{OH} / \mathrm{CH}_{3}\right)$, and $(\mathrm{O}=\mathrm{C}-\mathrm{H} / \mathrm{C}-\mathrm{H})$ pairs to represent hydroxyl, ketonic, carboxyl, and carbonyl oxygen functional groups as produced from a complete water dissociation, in accordance to the following reactions:

$\begin{array}{llll}\text { Hydroxyl: } & \mathrm{H}_{2} \mathrm{O}(\mathrm{l}) & +* \leftrightharpoons \mathrm{HO}^{*} & +\mathrm{H}^{+}(\mathrm{aq})+e^{-} \\ \text {Ketonic: } & \mathrm{H}_{2} \mathrm{O}(\mathrm{l}) & +* \leftrightharpoons \mathrm{O}^{*} & +2 \mathrm{H}^{+}(\mathrm{aq})+2 e^{-} \\ & & & \\ \text {Carboxyl: } & 2 \mathrm{H}_{2} \mathrm{O}(\mathrm{l}) & +* \leftrightharpoons \mathrm{O}^{*} \mathrm{OH} & +3 \mathrm{H}^{+}(\mathrm{aq})+3 e^{-} \\ & & +* \leftrightharpoons \mathrm{O}^{*} \mathrm{H} & +\mathrm{H}^{+}(\mathrm{aq})+e^{-}\end{array}$

where * denotes a graphite surface site and $\mathrm{X}^{*}$ denotes the generalized adsorption of oxygen functional groups species on the surface. [34]

The Gibbs free energy is defined by the following equation:

$$
G=U+p V-T S
$$

where $G$ is the Gibbs Free Energy of a thermodynamic system, which is related to the internal energy $(U)$ and the entropy $(S)$ of the system, while $p, V$, and $T$ denote the pressure, volume, and temperature of the system of interest. 
For calculations that involve solids, the $p V$ term was kept constant by fixing the size of the simulation box. However, the change in pressure and volume are negligible for solids, which simplifies the Gibbs free energy into:

$$
G=U-T S
$$

The internal energy is obtained by taking DFT energy calculations $\left(E_{D F T}\right)$ and including the thermal correction in the form of zero-point energy (ZPE), which changes the equation into:

$$
G=E_{D F T}+Z P E-T S
$$

Separate calculations of $G$ for * $\mathrm{n}_{2} \mathrm{O}$, and $\mathrm{X}^{*}$ allow for the change in the Gibbs free energy due to the adsorption of oxygen functional groups $(\Delta G)$, that is defined as:

$$
\Delta G=G\left(\mathrm{X}^{*}\right)-G\left({ }^{*}\right)-G\left(\mathrm{n}_{2} \mathrm{O}\right)
$$

All species formed through the splitting of water molecules as defined by reactions 1(a-d) were placed onto graphite surfaces. Proton chemisorption was modeled by the formation of C-H bonds with graphite surfaces in the cases of hydroxyl, ketonic, and carbonyl groups. In the case of carboxyl groups however, protons were adsorbed onto the surface through the formation of $-\mathrm{CH}_{3}$ group with a surface carbon atom to maintain the stoichiometry of the reaction. Although we also introduced H-terminated surfaces into all of the oxygenated surface group systems that we considered, we do not expect these H-terminations to affect our results, as fully H-terminated surface has been proven both experimentally and computationally to be inactive towards interfacial reactions in LIBs. [21,35-37]

To determine the relative free energy for each species in a chemical reaction, this value needs to be established against a reference cell, which is the standard hydrogen electrode (SHE). At standard conditions of $\mathrm{pH}=0$ and $p(\mathrm{H} 2)=1$ bar, the SHE reaction:

$$
\mathrm{H}^{+}(\mathrm{aq})+e^{-} \leftrightharpoons 1 / 2 \mathrm{H}_{2}(\mathrm{~g})
$$

has $\Delta G^{\circ}=0$ at $298 \mathrm{~K}$. The electrical potential $(U)$ for this reaction is set as: 


$$
U_{\text {SHE }}=0 \mathrm{~V}
$$

At equilibrium, the forward and reverse rates of reaction (3) are equal. The reverse reaction is termed the reversible hydrogen electrode (RHE). Extrapolation of the same equilibrium argument towards the electrical potential [24] results in $U_{\mathrm{RHE}}$ :

$$
U_{\mathrm{RHE}}=U_{\mathrm{SHE}}=0 \mathrm{~V}
$$

The value of $U_{\mathrm{RHE}}=0 \mathrm{~V}$ applies at both $\mathrm{pH}=0$ and when in equilibrium with SHE at all conditions.

However, changes in the $\mathrm{pH}$ in which the reaction takes place also changes the electric potential of the system, which in turn affects the adsorption energies of the adsorbates. The relationships between the $\mathrm{pH}$ of a system and both $U_{\mathrm{RHE}}$ and $\mathrm{U}_{\mathrm{SHE}}$ is defined in accordance with the following equation:

$$
U_{\mathrm{RHE}}=U_{\mathrm{SHE}}+\frac{k_{B} T(\ln 10) p H}{e}
$$

where the $\left(k_{\mathrm{B}} T(\ln 10) \mathrm{pH}\right)$ term describes the free energy in terms of $\mathrm{H}^{+}$concentration, and $e$ is the charge of the adsorbates. Combining these relationships, the Gibbs free energy for reaction (3) can be written as:

$$
\Delta G=e U_{\mathrm{RHE}}=e U_{\mathrm{SHE}}+k_{\mathrm{B}} T(\ln 10) \mathrm{pH}
$$

At $298 \mathrm{~K}$ (RTP), the surface is assumed to be in equilibrium with liquid water. Therefore, the extent of oxidation of water molecules and adsorption of various species on graphite can be determined by relating $\mathrm{U}$ and $\mathrm{pH}$ through the chemical potential of $\mathrm{H}^{+}$and $\mathrm{e}^{-}$. The assumption that reaction (3) is in equilibrium so that $U=0 \mathrm{~V}$ for both $U_{\mathrm{SHE}}$ and $U_{\mathrm{RHE}}$ at $\mathrm{pH}=0$ and $p\left(\mathrm{H}_{2}\right)=1$ bar, permits formulating the reactions of oxygen functional groups for surface adsorption in $1(\mathrm{a}-\mathrm{d})$ as:

$$
\begin{aligned}
& \text { Hydroxyl: } \mathrm{H}_{2} \mathrm{O}(\mathrm{l}) \quad+* \leftrightharpoons \mathrm{HO}^{*} \quad+1 / 2 \mathrm{H}_{2}(\mathrm{~g}) \\
& \text { Ketonic: } \mathrm{H}_{2} \mathrm{O}(\mathrm{l}) \quad+* \leftrightharpoons \mathrm{O}^{*}+\mathrm{H}_{2}(\mathrm{~g}) \\
& \text { Carboxyl: } 2 \mathrm{H}_{2} \mathrm{O}(\mathrm{l}) \quad+* \leftrightharpoons \mathrm{O}^{*} \mathrm{OH} \quad+3 / 2 \mathrm{H}_{2}(\mathrm{~g})
\end{aligned}
$$




$$
\text { Carbonyl: } \mathrm{H}_{2} \mathrm{O}(\mathrm{l}) \quad+* \leftrightharpoons \mathrm{O} * \mathrm{H} \quad+1 / 2 \mathrm{H}_{2}(\mathrm{~g})
$$

where the adsorption of hydrogen atoms is treated as adsorption of gaseous species rather than of protons. [24] The adsorption of molecular hydrogen molecules $\left(\mathrm{H}_{2}(\mathrm{~g})\right)$ are treated as spontaneous events, [38,39] and the change in the energies due to $\mathrm{H}_{2}(\mathrm{~g})$ molecules adsorbing on graphite are assumed to contribute a negligible amount to the overall energies.

By combining equations (2d) and (5), the Gibbs free energy for oxygen group adsorptions on the graphite surface $G\left(\mathrm{X}^{*}\right)$ at any $\mathrm{pH}$ and potential with $p\left(\mathrm{H}_{2}\right)=1$ bar can then be calculated as a function of potential $\mathrm{U}_{\mathrm{SHE}}$ and $\mathrm{pH}$ :

$$
\Delta G\left(\mathrm{X}^{*}\right)=\Delta G_{0}\left(\mathrm{X}^{*}\right)-e U_{\mathrm{SHE}}-k_{\mathrm{B}} T(\ln 10) \mathrm{pH}
$$

or in terms of RHE:

$$
\Delta \mathrm{G}\left(\mathrm{X}^{*}\right)=\Delta G_{0}\left(\mathrm{X}^{*}\right)-e U_{\mathrm{RHE}}
$$

where $\Delta G_{0}\left(\mathrm{X}^{*}\right)$ is the Gibbs free energy for oxygen adsorption at $\mathrm{pH}=0$ and $p\left(\mathrm{H}_{2}\right)=1$ bar, while the $\left(k_{\mathrm{B}} T\right.$ $(\ln 10) \mathrm{pH})$ term corrects the free energy of $\mathrm{H}^{+}$by the concentration dependence to the entropy. In the construction of the surface Pourbaix diagram, the contribution of electric field ( $\left.\Delta G_{\text {field }}\right)$ into $\mathrm{G}\left(\mathrm{X}^{*}\right)$ is usually ignored. [40]

The free energies $\left(\mathrm{G}\left(\mathrm{X}^{*}\right)\right)$ of graphite surfaces with a specific oxygen functional group at $\mathrm{pH}=0$ are plotted against $U_{\mathrm{RHE}}$ for different coverages to determine the composition and the surface structure at a given $U_{\text {RHE. }}$ For each oxygenated surface group, the coverage with the lowest $G\left(X^{*}\right)$ corresponds to the most stable surface. Surface Pourbaix diagrams are then constructed by plotting $U_{\text {RHE }}$ for different surface coverages over a range of $\mathrm{pH}$. Based on equation (7a), graphite surfaces with different surface coverages are shown on surface Pourbaix diagrams as lines with a slope of $\frac{-k_{B} T(\ln 10)}{e}$. Our method for constructing surface Pourbaix diagrams for the adsorption of different oxygen functional groups on graphite as a function 
of surface coverage is an adaptation from a very similar method that is generally used for analyzing water splitting reactions. [41]

\section{Results and Discussion}

\section{1 (11̄̄o) edge surface}

\subsubsection{Preparation of the surface}

The $(11 \overline{2} 0)$ edge surface has 48 surface carbon atoms within the simulation cell we used. Oxygen functional groups are placed onto the $(11 \overline{2} 0)$ pristine graphite surface in increments of $1 / 8$ of the total surface carbon atoms until all of the surface carbon atoms are covered by oxygen functional groups and hydrogen atoms in accordance with equations 6 (a-d) for hydroxyl, ketonic, and carbonyl groups, respectively. 24,16 , and 24 water molecules are required to attain $100 \%$ surface coverage for hydroxyl, ketonic, and carbonyl groups, respectively. Splitting water molecules into oxygen functional groups in accordance with equations 6(a-d) also resulted in the formation of hydrogen $\left(\mathrm{H}_{2}(\mathrm{~g})\right)$ molecules. Hydrogen molecules are then manually adsorbed onto the graphite surface to bind with surface carbon atoms and form $\mathrm{C}-\mathrm{H}$ constituents in the cases of hydroxyl, ketonic, and carbonyl groups. Adsorption of $\mathrm{H}_{2}(\mathrm{~g})$ molecules onto graphite surfaces to create H-terminated surfaces are treated as spontaneous events, $[38,39]$ and the energy changes brought by $\mathrm{H}_{2}(\mathrm{~g})$ adsorption is assumed to be negligible. Furthermore, the introduction of H-termination into graphite systems with oxygenated surface groups is assumed to not affect the oxygenated graphite surface as H-termination is found to be inactive in LIBs. [21,35-37]

In reality, functionalization of graphite anode surfaces with oxygen functional groups increases the $d$ spacing of graphite, thus enabling faster lithium intercalation for faster charging. [11] However, due to limitations within the DFT computational scheme, in which the $d$-spacing between graphite layers and the size of simulation cell are fixed, simulating a graphite surfaces with $100 \%$ coverage of pure carboxyl functional is not achievable. DFT optimizations of graphite surfaces with $100 \%$ coverage of pure carboxyl 
groups results in the formation of gaseous species that exhibit artificial defects on the graphite surfaces. These artificial defects arise due to the distance between graphite interlayers $(\sim 3.35 \AA)$ being too short to accommodate carboxyl groups that have a length of $\sim 3.02 \AA$. The small gap left upon the adsorption of two carboxyl groups on two adjacent graphite layers is not enough to minimize the repulsive forces between the neighboring carboxyl groups of adjacent graphite layers. To minimize the repulsion, ketonic groups were introduced to provide sufficient gaps between carboxyl groups on the graphite surface. The carboxyl and ketonic groups were adsorbed on the surface of every other graphite layer in an alternating fashion, as shown in Figure 1a. Ketonic groups were chosen as filler in our simulation cells as ketonic groups are adsorbed on the graphite surface in atomic fashion as $\mathrm{C}=\mathrm{O} / 2 \mathrm{C}-\mathrm{H}$, thus eliminating competition for space with the carboxyl groups. Additionally, the presence of ketonic groups sandwiched between two layers of carboxyl groups was found to be essential to passivate the graphite layers to maintain the integrity and shape of graphite layers. In comparison, if carboxyl groups were only adsorbed on every other layer while the sandwiched layer were kept empty, carboxyl groups were found to bind to two adjacent graphite layers to form arches, as shown in Figure 1b. Therefore, we define the $100 \%$ coverage of the carboxyl system as the adsorption of carboxyl and ketonic groups on the graphite surface in a 1:1 ratio. The 1:1 ratio of carboxyl:ketonic functional groups that constitute the carboxyl system in this paper is obtained by manually splitting 32 water molecules, in which 24 water molecules are split into $12(\mathrm{OOH} / 3 \mathrm{H})$ pairs to represent carboxyl functionals group as shown in equation $1 \mathrm{c}$, and 8 water molecules that are adsorbed as $(\mathrm{O} / \mathrm{H} / \mathrm{H})$ ketonic functional groups (equation 1b). The hydrogen atoms in the carboxyl system are manually adsorbed to the graphite surface to bind with the surface carbon as $\mathrm{CH}_{3}$ instead to maintain the correct stoichiometry of the water molecules in the cell. 
a)

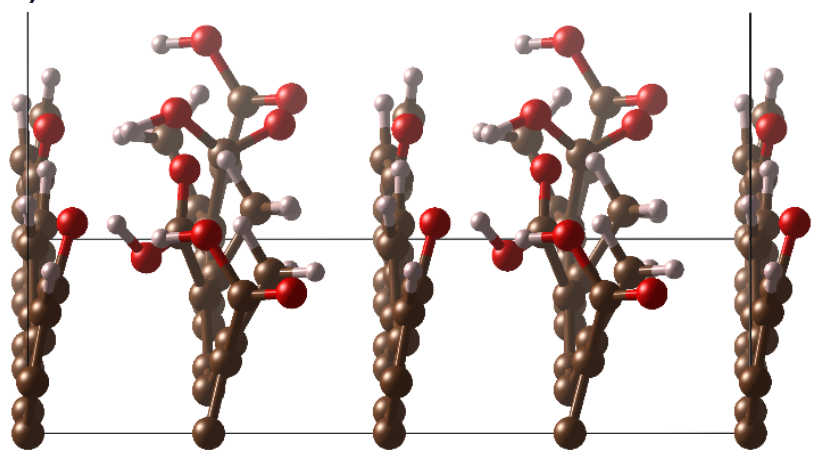

b)

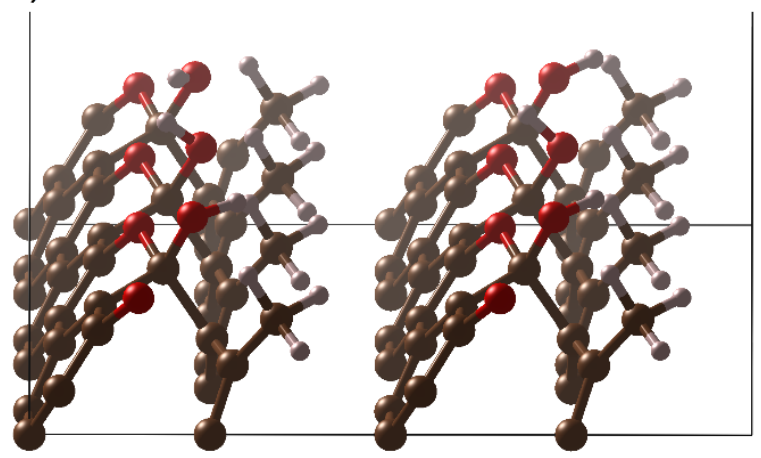

Figure 1: a) Our carboxyl system that is composed of carboxyl and ketonic groups in a 1:1 ratio, where carboxyl and ketonic groups are adsorbed on graphite in alternating layers to minimize repulsion between carboxyl groups of two adjacent graphite layers, and b) removal of ketonic groups in the alternating layers results in adjacent graphite layers forming arches that are capped by carboxyl groups. $\mathrm{C}, \mathrm{O}$, and $\mathrm{H}$ atoms are depicted in brown, red, and white, respectively.

\subsubsection{Generation of Pourbaix diagrams}

For all systems considered, the amount of adsorbed oxygen:hydrogen atoms are kept in a 1:2 ratio, to mimic the ratio of oxygen:hydrogen in water molecules. The effects of surface coverage on the stabilities of oxygen functional groups on the graphite surface, and concomitantly, the extent of surface coverage of each oxygenated functional is investigated through the calculation of the adsorption free energies $(\Delta G)$.

The $\Delta \mathrm{G}$ associated with the adsorption of oxygen functional groups on the graphite surface is reported in Table 1 as a function of surface coverage.

Table 1: The Gibbs free energies $(\Delta G)$ for adsorption of different oxygen functional groups on the graphite surface. $\Delta G$ is reported in $\mathrm{eV}$, while surface coverage (ML) is described as a fraction, where $8 / 8$ denotes $100 \%$ coverage of graphite surface by oxygen functional groups in the cases of hydroxyl, ketonic, and carbonyl. For carboxyl, the 8/8 ML describes the graphite surface that is fully populated by carboxyl and ketonic group in a 1:1 ratio.

\begin{tabular}{ccccccccc}
\hline \multirow{2}{*}{$\begin{array}{c}\text { Oxygen } \\
\text { groups }\end{array}$} & $\mathbf{7}$ Surface Coverage (ML) \\
\cline { 2 - 9 } & $\mathbf{8 / 8}$ & $\mathbf{7 / 8}$ & $\mathbf{6 / 8}$ & $\mathbf{5 / 8}$ & $\mathbf{4 / 8}$ & $\mathbf{3 / 8}$ & $\mathbf{2 / 8}$ & $\mathbf{1 / 8}$ \\
\hline Hydroxyl & -63.59 & -56.21 & -48.17 & -40.42 & -32.24 & -24.61 & -16.42 & -8.43 \\
\hline Ketonic & -61.98 & -54.70 & -47.41 & -40.09 & -32.87 & -24.68 & -16.48 & -8.26 \\
\hline Carboxyl & -23.38 & -20.73 & -17.67 & -22.18 & -14.15 & -8.27 & -14.54 & -9.98 \\
\hline Carbonyl & -22.78 & -23.90 & -18.51 & -18.17 & -12.14 & -11.63 & -7.60 & -6.66 \\
\hline
\end{tabular}


It can be seen in Table 1 that the Gibbs free energies $(\Delta \mathrm{G})$ are negative for all oxygen functional groups, regardless of the fractional surface coverage. The negative free energies imply that a bare graphite surface is very vulnerable to electron-accepting oxygen functional groups, which causes the adsorption of all oxygen functional groups on bare $(11 \overline{2} 0)$ graphite surface to be spontaneous over all surface coverages. Table 1 also shows a general inverse relationship between the values of $\Delta \mathrm{G}$ and the surface coverage of oxygen functional groups, in which the values of $\Delta \mathrm{G}$ across the same oxygen group become more negative with the population increase of oxygen groups on the graphite surface. For hydroxyl and ketonic functional groups, Table 1 shows that each $1 / 8$ increase in the surface coverage by these two functional groups is accompanied by, on average, about an $8 \mathrm{eV}$ decrease in $\Delta \mathrm{G}$ values.

Meanwhile, the values of $\Delta \mathrm{G}$ reported in Table 1 for carbonyl groups are seen to follow three distinctive trends: First, there is a general reciprocal relationship between values of $\Delta \mathrm{G}$ and surface population. Second, the incremental increases in surface coverage of carbonyl groups: $2 / 8 \mathrm{ML} \rightarrow 3 / 8 \mathrm{ML}, 4 / 8 \mathrm{ML} \rightarrow 5 / 8$, and $6 / 8 \mathrm{ML} \rightarrow 7 / 8 \mathrm{ML}$ are accompanied by much bigger differences in $\Delta \mathrm{G}$ values between the two data points involved in comparison to the less than $1 \mathrm{eV}$ differences in the $\Delta \mathrm{G}$ values of the incremental increases: $1 / 8$ $\mathrm{ML} \rightarrow 2 / 8 \mathrm{ML}, 3 / 8 \mathrm{ML} \rightarrow 4 / 8$, and 5/8 ML $\rightarrow 6 / 8 \mathrm{ML}$ as shown by the plot in Figure 2a. The nonlinear trend in $\Delta \mathrm{G}$ versus ML coverage is due to the presence of empty surface sites on graphite layers on which the carbonyl groups are adsorbed. For datapoints with large $\Delta \mathrm{G}$ value differences, at least one of the graphite layers in the systems are observed to consist of both carbonyl groups and empty surface sites. During DFT optimization, hydrogen atoms that belong to carbonyl groups leave the carbonyl group and move to the next available surface site on the same layer the carbonyl group is adsorbed on, depicted in Figure $2 \mathrm{~b}$. 

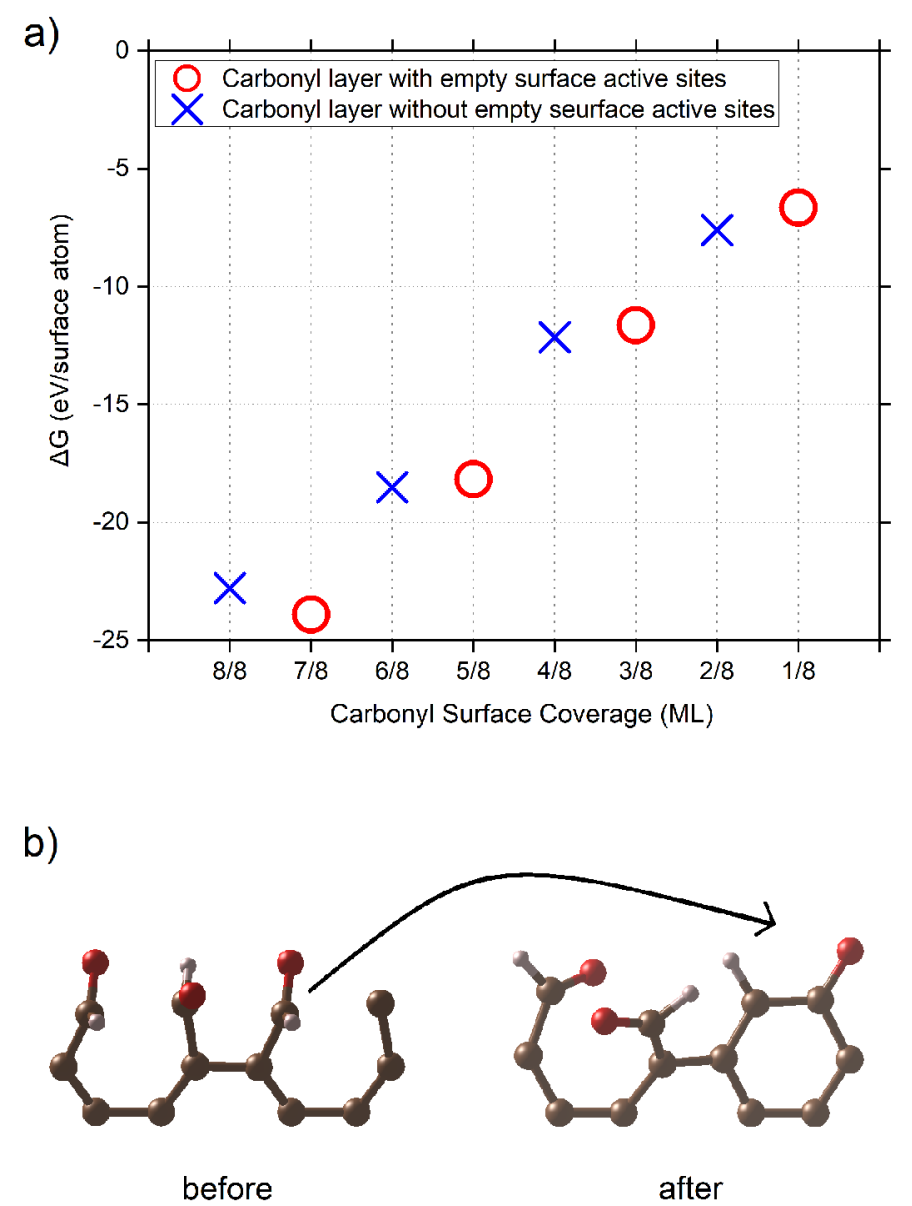

Figure 2: a) Changes in $\Delta G$ values as function of surface carbonyl groups coverage and b) the transformation of a carbonyl group into a ketonic group in the presence of an empty surface site on graphite layer that further stabilizes the graphite surface. $\mathrm{C}, \mathrm{O}$, and $\mathrm{H}$ atoms are depicted in brown, red, and white, respectively.

The abstraction of hydrogen by the empty surface sites transforms the carbonyl groups into ketonic groups, which brings further stabilization to the system. However, the transformation from carbonyl into ketonic group only occurs when the empty site is located in the same graphite layer with the carbonyl group. The functional group transformation will not happen if the empty sites are located on different graphite layers, as the distance between graphite layers is much greater than the distance between two carbon atoms of the same layer (distance between two graphite layers is $\sim 3.35 \AA$, while the distance between two carbon atoms on the surface of the same graphite layer is $\sim 1.42 \AA$ ). [42] Finally, it can also be seen in Table 1 that for carbonyl groups, the decrease in the values of $\Delta \mathrm{G}$ become less prevalent with the increase in surface 
coverage. This suggests that the as the graphite surface becomes more populated by carbonyl groups, the graphite loses its sensitivity towards the amounts of adsorbed carbonyl groups.

Furthermore, we would also like to point out that the $\Delta \mathrm{G}$ values in Table 1 show that the $\Delta \mathrm{G}$ value for the carbonyl system with $8 / 8$ surface coverage is less negative than the $\Delta \mathrm{G}$ value for $7 / 8$ surface coverage. It thus implies that the carbonyl system with $8 / 8 \mathrm{ML}$ surface coverage is less stable than the $7 / 8 \mathrm{ML}$. Although comparison between the $\Delta \mathrm{G}$ values of carbonyl at $8 / 8 \mathrm{ML}$ and $7 / 8 \mathrm{ML}$ seems to contradict the general trend of inverse relationship between $\Delta \mathrm{G}$ values and functional groups surface population, it can be explained through the combination of the effect of both functional group transformation from carbonyl to ketonic group and the decrease in the difference of $\Delta \mathrm{G}$ values that get smaller as surface population increases that is observed in the carbonyl system, as previously discussed. The $8 / 8 \mathrm{ML}$ has $100 \%$ surface coverage which means that there is no empty site to allow for functional group transformation from carbonyl to ketonic to stabilize the system and brings down its $\Delta \mathrm{G}$ value, while the $7 / 8 \mathrm{ML}$ system benefits from the presence of the empty surface sites. Additionally, the differences in the $\Delta \mathrm{G}$ values between datapoints $1 / 8$ $\mathrm{ML} \rightarrow 2 / 8 \mathrm{ML}, 3 / 8 \mathrm{ML} \rightarrow 4 / 8$, and 5/8 $\mathrm{ML} \rightarrow 6 / 8 \mathrm{ML}$ get smaller as the carbonyl population on the surface increases. The difference in the $\Delta \mathrm{G}$ values as the surface coverage increases from $1 / 8 \mathrm{ML}$ to $2 / 8 \mathrm{ML}$ is 0.94 $\mathrm{eV}$, which reduces to $0.51 \mathrm{eV}$ and $0.34 \mathrm{eV}$ for increases from $3 / 8 \mathrm{ML}$ to $4 / 8$, and $5 / 8 \mathrm{ML}$ to $6 / 8 \mathrm{ML}$, respectively. Based on these values, it is logical to expect that if the 7/8 ML does not have empty surface sites for functional group transformations to happen, the difference in the $\Delta \mathrm{G}$ values between $7 / 8 \mathrm{ML}$ and 8/8 ML would be even smaller.

In the model system we are using, each graphite layer consists of 6 surface carbon atoms, which means a maximum of $3(\mathrm{OOH} / 3 \mathrm{H})$ carboxyl group pairs can be adsorbed on a single graphite layer to saturate the layer. Carboxyl layers are sandwiched by a ketonic layer that are absorbed in $(\mathrm{O} / \mathrm{H} / \mathrm{H})$ configuration as shown in Figure 1a to minimize repulsions between carboxyl groups of adjacent layers. Only two ketonic groups are required to attain a fully saturated graphite layer. Full saturation of at least one graphite layer by carboxyl groups are achieved at 2/8 ML, 4/8 ML, and 8/8 ML surface coverage as shown in Table 2. On 
the other hand, whenever a ketonic layer is introduced, the ketonic layers are kept fully saturated to minimize its effect in the $\Delta \mathrm{G}$ evaluation of carboxyl groups. The compositions of carboxyl and ketonic functional groups that we employ to make up our carboxyl systems at each surface coverage along with the indication of whether the carboxyl layers are fully saturated are given in Table 2. Note that two water molecules are required to form a carboxyl group.

Table 2: Composition of carboxyl and ketonic groups at each surface coverage and whether the graphite layers are fully saturated by carboxyl layers. On the other hand, a graphite layer in our computational model can only adsorb two ketonic groups. Ketonic layers that sandwich two carboxyl layers are kept fully saturated at all surface coverages to minimize the effect of ketonic groups in the evaluation of $\Delta G$.

\begin{tabular}{cccccc}
\hline $\begin{array}{c}\text { Surface } \\
\text { Coverage (ML) }\end{array}$ & No. $\mathbf{H}_{\mathbf{2}} \mathbf{O}$ & $\begin{array}{c}\text { No. of } \\
\text { carboxyl } \\
\text { groups }\end{array}$ & $\begin{array}{c}\text { No. of ketonic } \\
\text { groups }\end{array}$ & $\begin{array}{c}\text { Full carboxyl } \\
\text { layer }\end{array}$ & $\begin{array}{c}\text { Full ketonic } \\
\text { layer }\end{array}$ \\
\hline $1 / 8$ & 4 & 1 & 2 & No & Yes \\
\hline $2 / 8$ & 8 & 3 & 2 & Yes & Yes \\
\hline $3 / 8$ & 12 & 5 & 2 & No & Yes \\
\hline $4 / 8$ & 16 & 6 & 4 & Yes & Yes \\
\hline $5 / 8$ & 20 & 7 & 6 & No & Yes \\
\hline $6 / 8$ & 24 & 8 & 8 & No & Yes \\
\hline $7 / 8$ & 28 & 10 & 8 & Yes & Yes \\
\hline $8 / 8$ & 32 & 12 & 8 & & \\
\hline
\end{tabular}

Table 2 shows that two ketonic groups are introduced to make up a ketonic layer for 1/8 ML, 2/8 ML, and 3/8 ML surface coverages. As the number of ketonic groups are the same in the $1 / 8 \mathrm{ML}, 2 / 8 \mathrm{ML}$, and $3 / 8$ ML systems, straight comparison of $\Delta \mathrm{G}$ values between the three systems may be made. The $\Delta \mathrm{G}$ values presented in Table 1 show that the $\Delta \mathrm{G}$ value of $2 / 8 \mathrm{ML}$, with a full strip of carboxyl layer, has the most negative $\Delta \mathrm{G}$ out of these three surfaces. The same trend can be seen for $6 / 8 \mathrm{ML}, 7 / 8 \mathrm{ML}$, and $8 / 8 \mathrm{ML}$ surface coverages, where the most negative $\Delta \mathrm{G}$ value belongs to the $8 / 8 \mathrm{ML}$, which does not have empty sites on carboxyl layers. These results imply that a fully saturated carboxyl layer stabilizes the carboxyl system, which is the opposite effect from the trend observed for the carbonyl surfaces.

The difference in the effect brought by carbonyl and carboxyl functional groups on graphite surface stabilization may be explained by the difference in which these two functional groups attract electrons: 
carbonyl is an electron donating group, while carboxyl is an electron withdrawing group. [43] As a consequence, contrasting behaviors are observed regarding the full layer adsorption of these two functional groups on graphite. At the edge surface of graphite, the delocalized electrons in graphite are broken into dangling bonds that results in negative partial charges at the surface. The adsorption of electron withdrawing carboxyl groups helps stabilizes the surface by taking the partial charges away from the graphite surface. As the result, a graphite layer with full carboxyl groups adsorption is more stable than those that are not. On the other hand, the adsorption of electron donating carbonyl groups leads to the accumulation of negative partial charge at the surface. The accumulated partial charge at graphite surface further destabilizes the system, which explains why graphite layer with empty active sites is more stable than the graphite layers where the active sites are fully occupied by carbonyl groups.

To compare the adsorption $\Delta \mathrm{G}$ across different oxygen functional groups, normalization against the number of water molecules is required due to the different amount of water molecules involved at any given fraction of surface coverage. Direct comparison can be made between the $\Delta \mathrm{G}$ of hydroxyl and carbonyl groups, as both systems have the same number of water molecules. In the case of hydroxyl $v s$. carbonyl, it can be seen from Table 1 that the $\Delta \mathrm{G}$ of the hydroxyl group is more negative than that of the carbonyl for all surface coverages. It thus implies that the adsorption of hydroxyl groups on $(11 \overline{2} 0)$ graphite surface is much stronger than the carbonyls. Meanwhile, comparison with the carboxyl group requires the amount of ketonic groups that are present as fillers to be taken into consideration. In an acidic solution $(\mathrm{pH}=0), \Delta \mathrm{G}$ values for oxygen functional group adsorptions are $-5.62,-8.26,-1.72,-4.44 \mathrm{eV}$ per two water molecules for hydroxyl, ketonic, carboxyl, and carbonyl groups, respectively. The $\Delta \mathrm{G}$ value for carboxyl is obtained by taking the calculated $\Delta \mathrm{G}$ value for carboxyl at $1 / 8 \mathrm{ML}$ subtracted by the $\Delta \mathrm{G}$ value that comes from the adsorption of two ketonic groups to cancel the effect of two ketonic groups that are present in our 1/8 ML carboxyl system. We chose two water molecules as the normalization base point as it is the minimum amount of water molecules required to form a carboxyl group. 
In general, the normalized values of our results from Table 1 show that the adsorption strength of oxygen functional groups on $(11 \overline{2} 0)$ graphite edge are in the following order: carboxyl < carbonyl < hydroxyl $<$ ketonic. We found that the adsorption sites of oxygen functional groups on graphite or the fashion in which they are added onto the surface do not affect the overall $\Delta \mathrm{G}$ and its stability trends. The higher stability of ketonic and hydroxyl groups than carboxyl and carbonyl groups of the same coverage on the surface of $(11 \overline{2} 0)$ graphite are also observed on other surfaces, e.g., boron nitrides [44] and MXenes. [44] We also found that the $\Delta \mathrm{G}$ of hydroxyl and carboxyl adsorption on (112̄0) graphite follows the general scaling relation of the adsorption of the two oxygen functional groups on various surfaces, where $\Delta \mathrm{G} *_{\mathrm{OOH}}$ $=\Delta \mathrm{G} *_{\mathrm{OH}}+3.2 \mathrm{~V}( \pm 0.02 \mathrm{~V}) .[45,46]$ Both $\Delta \mathrm{G}$ and the strength of adsorption of oxygen functional groups on the surface of graphite therefore indicates the surface of $(11 \overline{2} 0)$ graphite will have high coverage of hydroxyl and ketonic groups. Furthermore, the adsorbed carboxyl and carbonyl groups on the edge sites of $(1 \overline{2} 0)$ graphite may be further reduced into ketonic and hydroxyl groups, [47] which also adds into the high amount of surface hydroxyl and ketonic functional groups. The most stable graphite surfaces for hydroxyl, ketonic, carboxyl, and carbonyl groups at $\mathrm{pH}=0$ over a range of surface coverages are shown in Figure 3. 
a) Hydroxyl

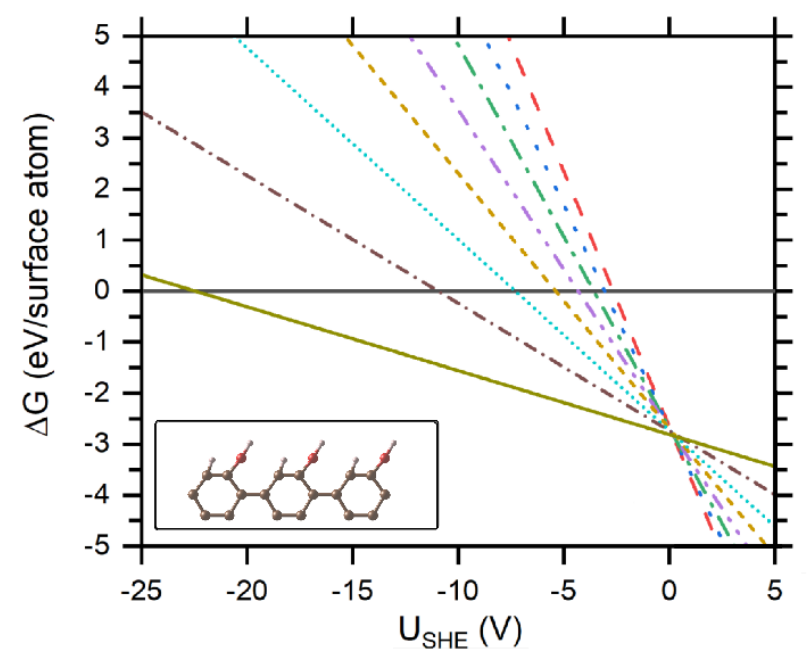

c) Carboxyl

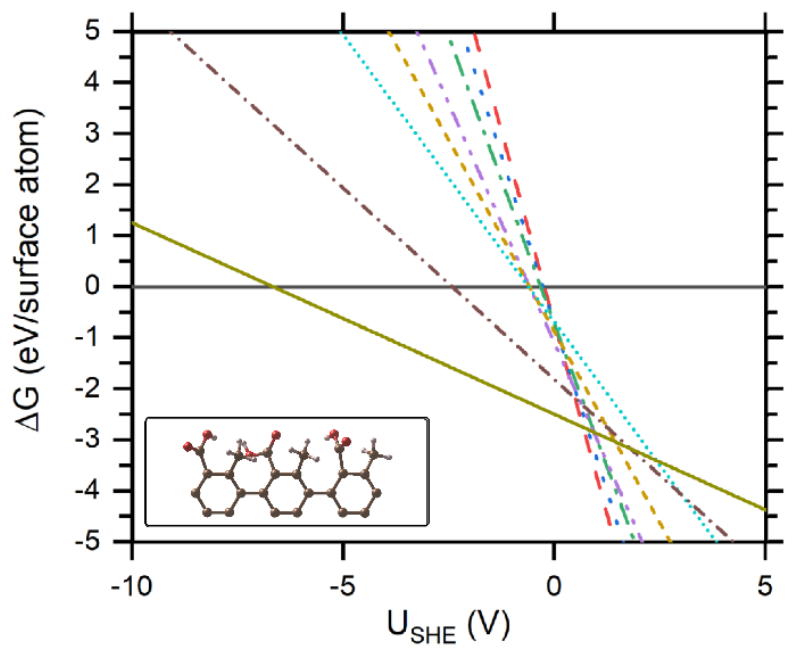

b) Ketonic

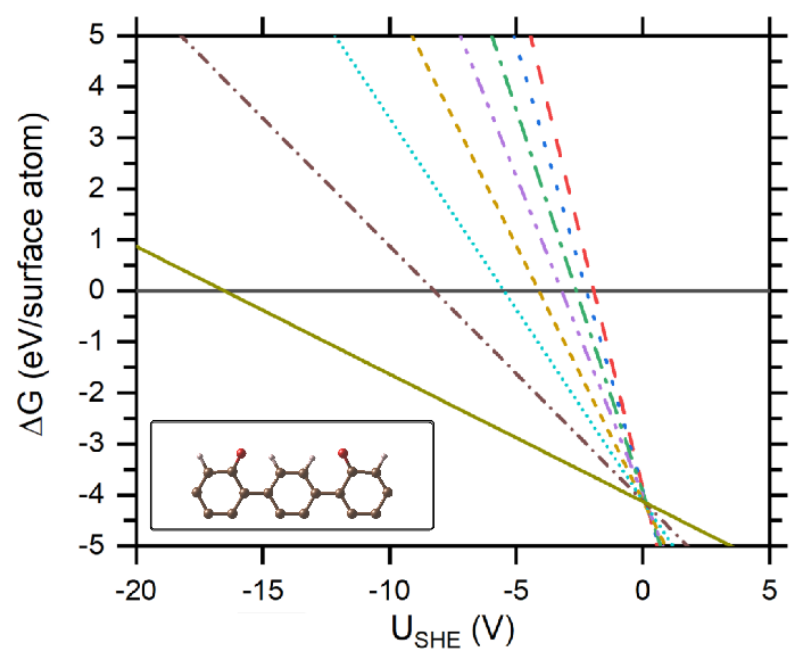

d) Carbonyl

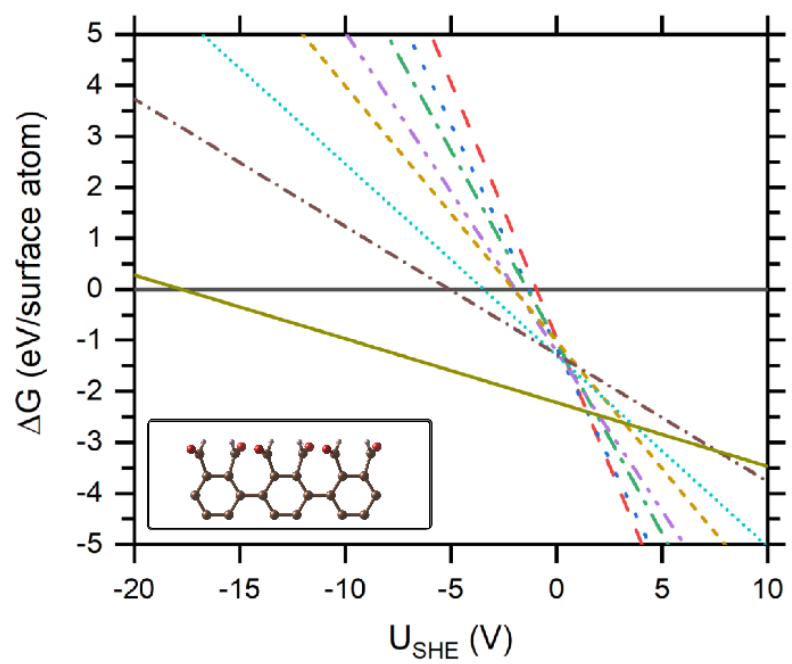

Oxygen Functionals Surface Coverage (ML):

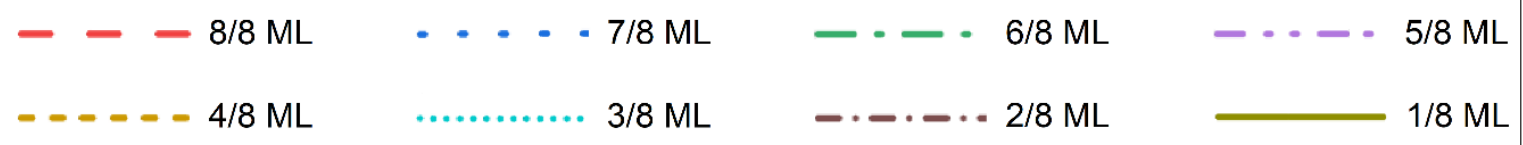

Pristine Edge Graphite (11̄ㅇ)

Figure 3: The most stable graphite surfaces for a) hydroxyl, b) ketonic, c) carboxyl, and d) carbonyl at $\mathrm{pH}=0$ over a range of surface coverages (denoted as ML). $\mathrm{C}, \mathrm{O}$, and $\mathrm{H}$ atoms are depicted in brown, red, and white, respectively. 
Figure 3 shows that the $\mathrm{U}_{\mathrm{SHE}}$ is the most negative at 1/8 ML for all oxygen functional groups studied. This result therefore indicates that at $\mathrm{pH}=0$, the most stable graphite surfaces are those that are decorated by the fewest surface oxygen functional groups. Across all oxygen functional groups, at 1/8 ML coverage and under standard conditions, hydroxyl is the most stable oxygen group adsorbed on the graphite surface as the $U_{\text {SHE }}$ of hydroxyl is the most negative. The negative $U_{\text {SHE }}$ values that are as low as $-22.47,-16.52$, 6.65 , and $-17.75 \mathrm{~V}$ are required to protect the graphite surface from being oxidized by hydroxyl, ketonic, carboxyl, and carbonyl groups, respectively. Above the potential that is required for protection of bare graphite (above $-22.47 \mathrm{~V}$ ), oxidation of water molecules takes place, and at least $1 / 8$ of the graphite surface will be covered by hydroxyl. The $\Delta \mathrm{G}$ for the adsorption of oxygen functional groups adsorption decreases even further with the increase in applied potential.

Upon application of a very small positive external potential, the coverage of oxygen functional groups on graphite surfaces are found to increase dramatically. The point of intersection between 1/8 ML lines with the 8/8 ML lines in Figure 3 also correspond to the amount of external potential required to change the surface coverage for functional group from $1 / 8 \mathrm{ML}$ to $8 / 8 \mathrm{ML}$. Therefore, the amount of external potential required to change the surface coverage from $1 / 8 \mathrm{ML}$ to $8 / 8 \mathrm{ML}$ for hydroxyl, ketonic, carboxyl, and carbonyl are: $0.2 \mathrm{~V}, 0.15 \mathrm{~V}, 0.67 \mathrm{~V}$, and $1.45 \mathrm{~V}$, respectively. Meanwhile, an applied external potential of $1.23 \mathrm{~V}\left(\mathrm{U}_{\mathrm{RHE}}\right)$ is required for water oxidation reactions to take place spontaneously. Seeing that the amount of applied external potential required for water oxidation reactions to occur is higher than the $\mathrm{U}_{\mathrm{SHE}}$ of hydroxyl, ketonic, and carboxyl at $8 / 8 \mathrm{ML}$, it can be concluded that these oxygen functional groups will have full surface coverages on $(11 \overline{2} 0)$ graphite. In contrast, carbonyl groups will only occupy $1 / 8$ of the $(11 \overline{2} 0)$ graphite surface.

Adsorption of oxygen functional groups on graphite transforms the $\mathrm{sp}^{2}$ orbital of the surface carbon atoms into $\mathrm{sp}^{3}$ hybridization, which destroys the aromaticity of the surface carbon rings and results in destabilization of the graphite layers. The adsorption induced destabilization is a local effect that accumulates with the increase in oxygen group surface coverage. This relationship is manifested in Figure 
3, where it can be seen that for any given value of $\Delta \mathrm{G}$, the increase in surface coverage for all oxygen functional groups considered are accompanied by the increase in the amount of external potential required ( $\left.\mathrm{U}_{\mathrm{SHE}}\right)$. For example, at $\Delta \mathrm{G}=0 \mathrm{eV}, \sim 20 \mathrm{~V}$ of external potential ( $\left.\mathrm{U}_{\mathrm{SHE}}\right)$ is required to turn the hydroxyl surface from having $1 / 8 \mathrm{ML}$ of surface coverage to $8 / 8 \mathrm{ML}$ full surface coverage. The increase in USHE shows that the successive adsorption of oxygen functional groups at the surface of $(11 \overline{2} 0)$ edge graphite is an endergonic process relative to the initial adsorption.

Changes in the $\mathrm{pH}$ values also bring changes to the $\mathrm{U}_{\mathrm{SHE}}$, which in turn affect the overall $\Delta \mathrm{G}$ values and the adsorption energies of the oxygen functional groups. [47] According to equation 5a, the change in $\mathrm{pH}$ of a system with the same oxygen functional group and surface coverage shifts the $\mathrm{U}_{\mathrm{SHE}}$ by $\frac{-k_{B} T(\ln 10)}{e * p H}$, which is $\frac{-0.059 \mathrm{~V}}{e * p H}$. [41] The plot of $\mathrm{U}_{\mathrm{SHE}}$ over a range of $\mathrm{pH}$ for a system with the same surface coverage is called a surface Pourbaix diagram. At any given $\mathrm{pH}$, the most thermodynamically stable surface is indicated by the lowest $U_{\text {SHE. }}$ Figure 4 shows the surface Pourbaix diagrams for the adsorption of hydroxyl, ketonic, carboxyl, and carbonyl groups of different coverages on a (11 $\overline{2} 0)$ graphite surface. 
a) Hydroxyl

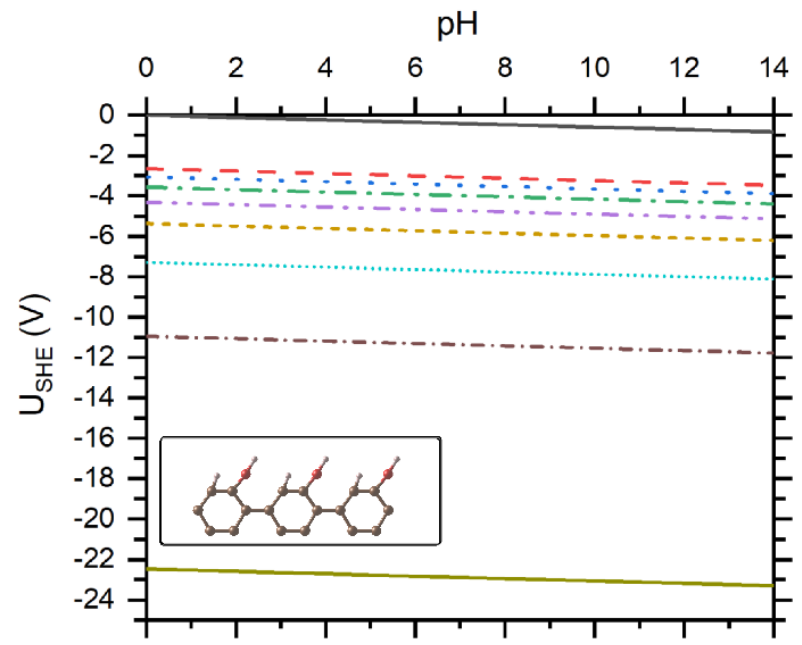

c) Carboxyl

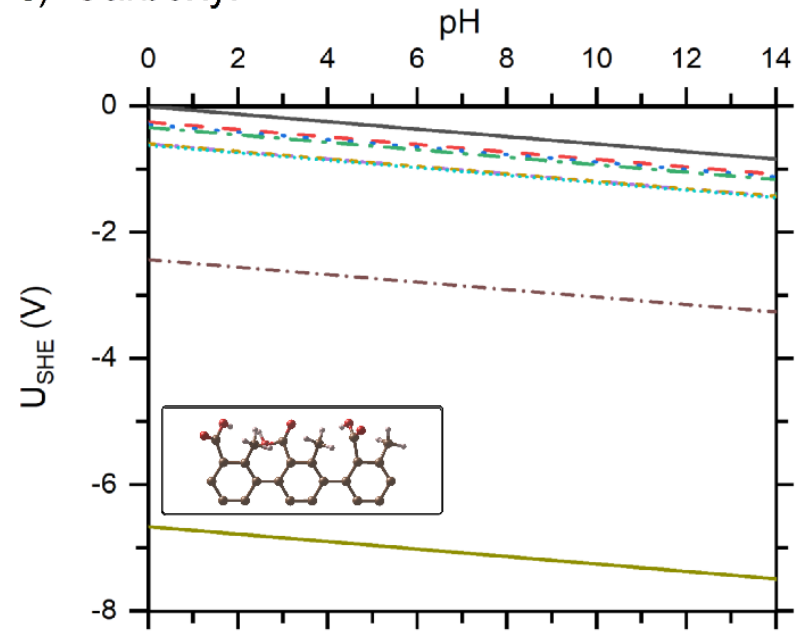

b) Ketonic

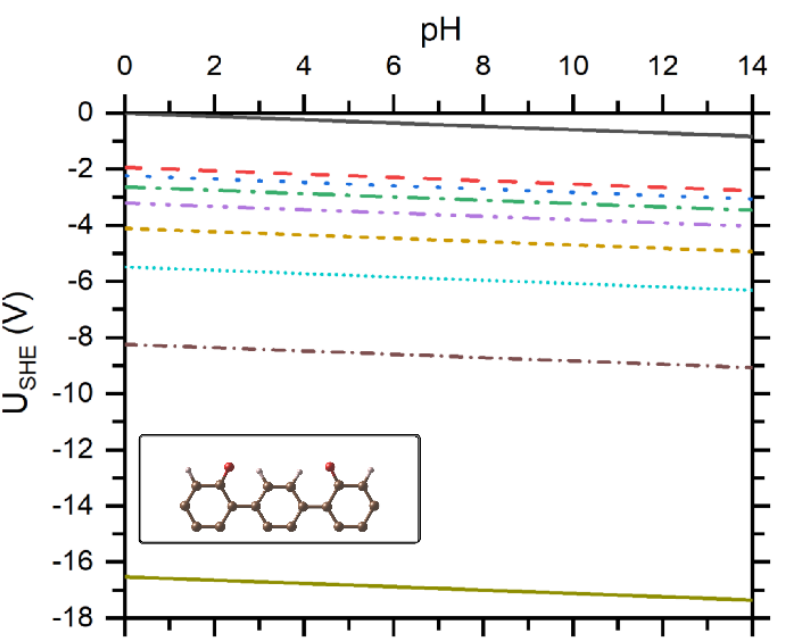

d) Carbonyl

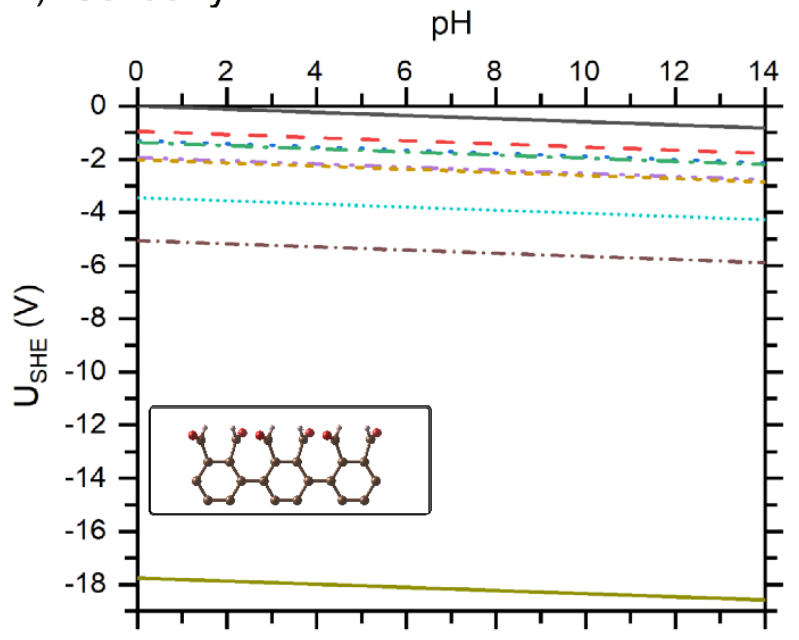

Oxygen Functionals Surface Coverage (ML):

$-\quad-8 / 8 \mathrm{ML}$

$\ldots-\ldots 4 / 8 \mathrm{ML}$
… $7 / 8 \mathrm{ML}$

$3 / 8 \mathrm{ML}$
- - $6 / 8 \mathrm{ML}$ $\ldots \ldots 5 / 8 \mathrm{ML}$

-.-.-.. 2/8ML $1 / 8 \mathrm{ML}$

Pristine Edge Graphite (11 $\overline{2} 0)$

Figure 4: Surface Pourbaix diagrams for a) hydroxyl, b) ketonic, c) carboxyl, and d) carbonyl oxygen functionalized graphite surfaces over a range of surface coverages (denoted as ML). $\mathrm{C}, \mathrm{O}$, and $H$ atoms are depicted in brown, red, and white, respectively.

As can be seen from Figure 4, the USHE of the adsorption of all oxygen functional groups with different surface coverages are negative for all $\mathrm{pH}$ values. Furthermore, the $\mathrm{U}_{\mathrm{SHE}}$ decreases as the $\mathrm{pH}$ increases, which indicates that the $\Delta \mathrm{G}$ associated with the adsorption energy of all oxygen functional groups also 
decreases as the $\mathrm{pH}$ moves from acidic to basic. As a result, the graphite surface would be even more prone to oxygen group attacks in basic solution. The increase in $\mathrm{pH}$ results in the stronger adsorption and further stabilization of oxygen functional groups on the surface of graphite and the graphite surface would be highly decorated in basic solution in comparison to acidic solution.

The Pourbaix diagrams in Figure 4 also highlight the difference in the ability of oxygen functional groups to affect the stability of the graphite surface. The discrete lines in the Pourbaix diagram for each calculated surface coverage for hydroxyl (Figure 4a) and ketonic (Figure 4b) functional groups indicate the sensitivity of the graphite surface to the amount of adsorbed hydroxyl and ketonic groups. This finding reveals the ability of both hydroxyl and ketonic groups to affect the stability of the graphite quite substantially. On the contrary, graphite is less sensitive towards the change in the quantity of adsorbed carboxyl and carbonyl groups. For carboxyl adsorption, Figure 4c shows that the lines for 3/8ML, 4/8 ML, $5 / 8 \mathrm{ML}$ and 6/8 ML lay on top of each other, thus indicating that the stability of graphite that is covered by 3/8ML, 4/8 ML, or 5/8 ML of carboxyl are similar. The same trend can also be seen for carbonyl functional groups in Figure 4d, where the surfaces with 4/8 ML and 5/8 ML are of similar stability, and the 6/8 ML surface is similar in stability to the $7 / 8 \mathrm{ML}$ carbonyl surface.

The operating $\mathrm{pH}$ of LIBs range between 7-14, subject to the materials of the electrodes, slurry components, and number of cycles. [48] In particular, the most commonly used anode in $\mathrm{LIBs}, \mathrm{LiC}_{6}$, have the near-neutral $\mathrm{pH}$ at the electrode/electrolyte at the beginning of the charge/discharge cycle. [49] Previous experimental studies have shown that the $\mathrm{pH}$ at this particular interface increases to a more basic region with the buildup of a solid-electrolyte interface (SEI) as the number of cycles increases. [50] Based on the surface Pourbaix diagrams of the oxygen functional groups shown in Figure 4, we conclude that at the operating temperature and $\mathrm{pH}$ of LIBs, the surface of $(11 \overline{2} 0)$ armchair graphite LIB anode will be fully covered by oxygenated functional groups. In particular, the oxygen functional groups at the graphite (11 $\overline{2} 0)$ surface would consist of a high fraction of hydroxyl and ketonic groups, while carboxyl and carbonyl groups will be present in a lower ratio. For example, under reaction conditions of $\mathrm{pH}=14$ and $\mathrm{U}_{\mathrm{SHE}}=-6.0 \mathrm{~V}$, the 
surface of $(11 \overline{2} 0)$ graphite will be composed of 4/8 ML hydroxyl, 3/8 ML ketonic, while carboxyl and carbonyl functional groups will make up the last $1 / 8 \mathrm{ML}$ of the graphite surface.

\section{2 (0001) basal surface}

As the basal sites of graphite in LIBs are also exposed to electrolyte/water/moisture, they may also be oxidized. However, due to the double bond in ketonic, carboxyl, and carbonyl functional groups, only hydroxyl functional groups are able to adsorb on pristine basal sites. We found that the adsorption of hydroxyl groups on the basal site of graphite is only possible in the dilute region, with the maximum adsorption occurring at 1/6 ML of surface coverage. In our model, the 1/6 ML hydroxyl coverage is manifested in the adsorption of $4 \mathrm{OH} / \mathrm{H}$ pairs on the basal sites of a single graphite layer that consists of 48

surface carbon atoms. Both the stability of hydroxyl group adsorption on basal graphite and its corresponding Pourbaix diagram are shown in Figure 5a and Figure 5b, respectively. 


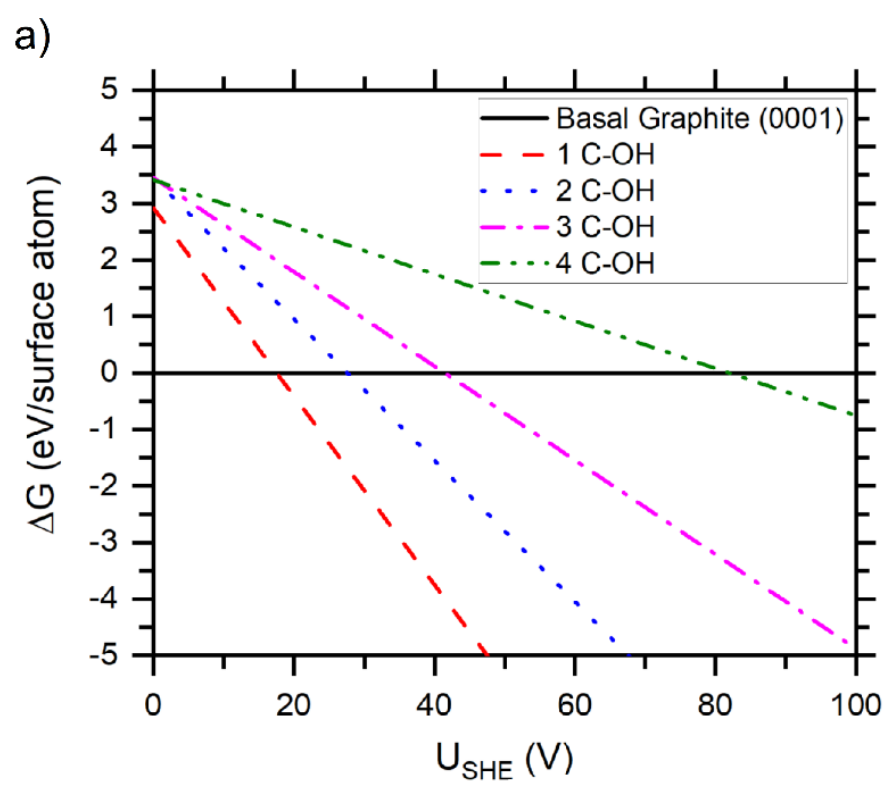

b)

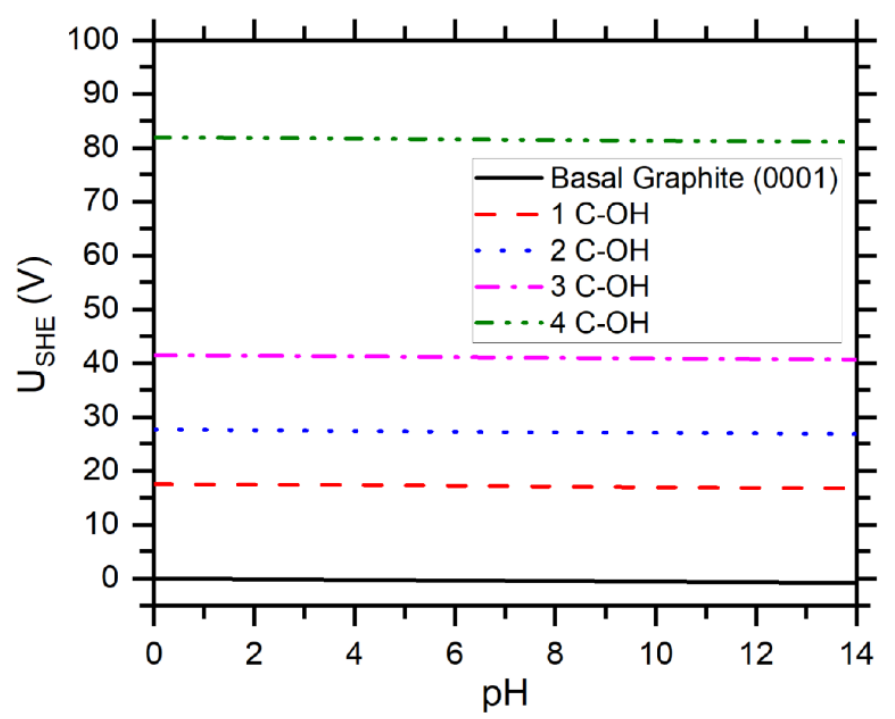

Figure 5: a) The stability of hydroxyl group adsorption on basal (0001) graphite at $\mathrm{pH}=0$ and b) the surface Pourbaix diagram for hydroxyl functionalized graphite surfaces.

Figure 5a shows that the adsorption of even one hydroxyl group is already a very endergonic process, in which an external potential of $\sim 20 \mathrm{~V}$ is required for the process to spontaneously occur. The increase in the $\mathrm{pH}$ does not have a significant effect in reducing the required external potential to assist the adsorption reaction, as shown in Figure 5b. As mentioned previously, though highly unfavorable, the basal surface of graphite (0001) is capable of holding up to 1/6 ML of hydroxyl groups. Based on the results presented in Figure 5 we conclude that the (0001) basal surface of the graphite anode will remain bare in LIBs. In LIBs, 
the inability of the basal surface to be oxidized ensures that the distance between graphite layers remains at least $\sim 3.35 \AA$, thus ensuring a smooth intercalation process of $\mathrm{Li}^{+}$ions.

Although highly unfavorable, up to four hydroxyl groups can be adsorbed on the (0001) basal surface of graphite to make up the $1 / 6 \mathrm{ML}$ of hydroxyl surface coverage. Coincidentally, our simulation cell is comprised of four carbon rings in the $x$-direction, and all four hydroxyl pairs are found to adsorb on the surface carbon atoms available in a row along the $x$-direction of our simulation cell. The -OH constituents of the hydroxyl groups are adsorbed in a meta- configuration to each other, while the $-\mathrm{H}$ constituents are found to adsorb at the ortho- locations in between two $-\mathrm{OH}$ constituents. The adsorption of the four $\mathrm{OH} / \mathrm{H}$ hydroxyl pairs on the basal sites of the (0001) graphite surface is shown in Figure 6a. The adsorption of the four $\mathrm{OH} / \mathrm{H}$ pairs in an orderly fashion on the basal sites of the (0001) graphite surface results in the clustering of the adsorption of $\mathrm{OH} / \mathrm{H}$ pairs. The clustering of the adsorption species on the basal sites leads to a more stable system with a lower total energy than if the adsorbed species were scattered on the basal surface, and is in accordance with previous computational studies. [51] However, as the consequence of the highly ordered clustered adsorption, the surface of the (0001) basal graphite becomes distorted, as shown in Figure 6b.

a)

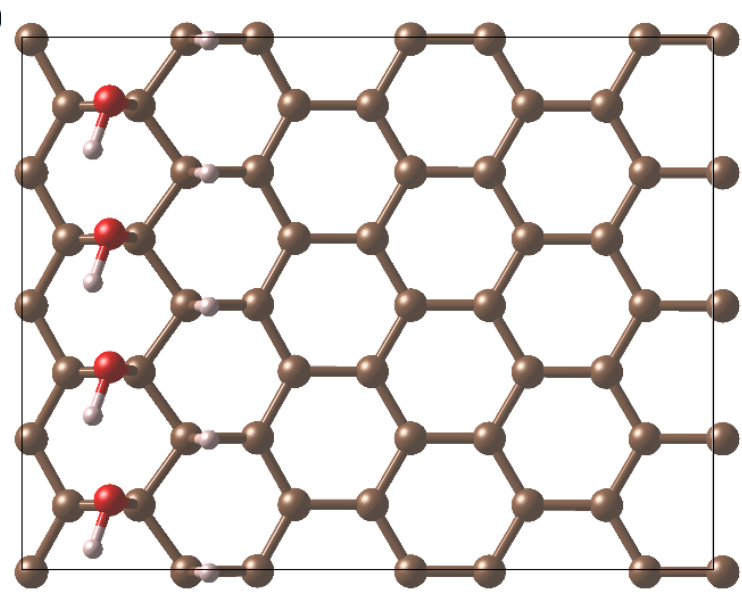

b)

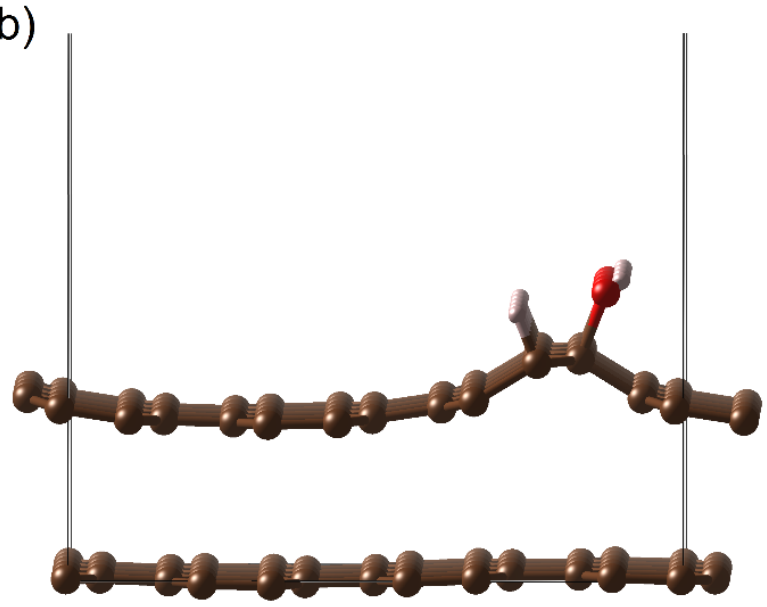

Figure 6: a) The top view of the adsorption sites of 4 hydroxyl groups on the (0001) basal graphite, in which the -OH constituents are adsorbed in the meta- configuration to each other, while the $-\mathrm{H}$ constituents are adsorbed at the ortho- sites in between two -OH groups. b) The distorted structure 
of (0001) basal graphite as a result of the highly ordered adsorption of the four hydroxyl groups. C, $O$, and $H$ atoms are depicted in brown, red, and white, respectively.

The distorted structure of the graphite surface has also been detected in experiments where the defects are known as "wrinkles". In the wrinkle sites, the graphite is consisted of two distinctive regions with two different C-C bond lengths: 1) aromatic regions, and 2) oxygen functional groups containing aliphatic 6membered rings. [52] The same distorted structure has also been observed on graphene, [14] in which the extent of deformation of the graphite structure by each oxygen group is quantified.

In addition to the aforementioned oxygen functional groups, epoxy (-O-) group adsorptions and $\mathrm{C}=\mathrm{C}$ double bond defects may also cause distortion to the graphite structure. Due to the vast combinations of the possible adsorption sites for both the epoxy and the hydrogen atoms in relation to the bridging epoxy group on the basal surface, the effect of epoxy group adsorption on graphite merits its own study. However, we do expect the adsorption of epoxy groups on the basal surface of graphite to also be in the dilute region with similar stability and behavior to those with hydroxyl functional groups that are shown here.

\subsection{Perspective on the Importance of Fine Tuning the Compositions of Oxygenated Functional Groups on} the Surface of Graphite Anode

The findings presented in this paper on the stability of different oxygenated functional groups on the surface of a graphitic anode and the surface structure and composition of the anode can be used to rationally guide further design and optimization of LIB materials. Surface oxygen functional groups substantially affect many of the chemical processes within an LIB, ranging from the formation of an SEI, to the behavior of binder materials and the efficacy of additives. The incorporation of oxygen functional groups on the surface of a graphite anode have been found to be beneficial to the performance of LIBs, as it increases the power density, charging rate, and life cycles of LIBs. [54] However, although commonly acknowledged, the literature on the differences in the effects brought by different oxygen functional groups on the aforementioned processes in LIBs is sparse. Furthermore, the current consensus in the battery community 
is that there is an ideal ratio range in which the effect of surface oxygen functional groups on the performance of an LIB is optimum. When the concentration of surface oxygen functional groups is outside of the optimum range, the effect is detrimental to the performance of LIBs: low concentration of surface oxygen functional groups results in low energy density and anode disintegration, while the concentration of surface oxygen group that is beyond the optimum range lead to an increase in irreversible charge loss. [55] To assist further advances in this field, we will give a quick overview on the effect of different surface oxygen functional groups on different chemical processes within an LIB and other electrochemical systems that also utilize graphite for one of their components, such as sodium-ion batteries (NIBs), lithium-ion hybrid supercapacitors (LICs), and fuel cells.

In LIBs, the interfacial reactions between electrolyte and graphite anode dictate the structures and properties of the SEI. We observe different effects brought by different surface oxygen functional groups on the density profile and dynamic behavior of electrolytes, which substantially affect the kinetics and growth rate of an SEI. [56] Our previous study has also reported that the type of surface oxygen groups at the edge sites of a graphitic anode determines the products of electrolyte decomposition reactions at the interface. [22] In particular, hydroxyl and ketonic rich surfaces are found to assist in additive decomposition on the anode that results in the formation of a thinner, more flexible, and more superior SEI. Meanwhile, high concentration of carboxyl and carbonyl functional group surfaces lead to higher rates of gaseous species formation, which may accumulate and result in battery explosion. [57] However, both carboxyl and carbonyl functional groups play an important role in improving the electrochemical properties of LIBs, as the presence of both carboxyl and carbonyl functional groups enhances the reversible capacities of LIBs, which leads to LIBs with higher energy density. [58] Furthermore, carboxyl and carbonyl groups improve the mechanical properties of a graphite anode as they allow binder materials to bind to the anode both covalently and through hydrogen bonding, which leads to higher adhesion to the anode. $[59,60]$ In comparison to hydroxyl and ketonic rich surfaces, carboxyl and carbonyl rich graphite anodes possess a more superior structural integrity, leading to LIBs with higher cyclabilities. [61] The competition between 
the stability and cyclability of LIBs in relation to the concentration of surface carboxyl and carbonyl groups shows the importance of fine tuning the graphite anode surface structure and composition so that the tradeoff between the two properties can be carefully controlled.

To further complicate the matter, the type and concentration of the surface oxygen functional groups at the basal surface also require careful consideration. A very small concentration of surface hydroxyl groups at the basal sites is necessary to support the architecture of the anode and increase the structural interlayer spacing and improve the charge rate of LIBs. However, high concentration of basal hydroxyl groups is detrimental to the charge rate of LIBs due to strong coulombic attractions between basal hydroxyl groups and the neighboring graphite layers, which reduces the interlayer spacing and slows down the charging rate. [62] Additionally, the concentration of epoxy groups at the basal sites needs to be minimized, as it lowers the initial coulomb efficiency of LIBs, which negatively impacts the electrochemical properties. [58]

Outside of LIBs, graphite can also be found on other electrochemical systems such as sodium-ion batteries (NIBs), lithium-ion hybrid supercapacitors (LICs), and fuel cells. The composition of oxygen functionalities at the surface of graphite in these systems also plays an important part in determining its performance. For example, in NIBs it is important that hydroxyl groups are adsorbed as clusters on graphene basal plane to avoid $\mathrm{NaOH}$ formation and phase separation. [63] In contrast to LIBs, the presence of epoxy groups is beneficial to the performance of NIBs as it acts as stable adsorption sites for sodium and enhances the adsorption energy. [64] On the other hand, the lifecycles of LICs are in direct correlation to the concentration of double bonds in the surface oxygen groups, as the double bonds in the surface oxygen groups induce lithium enolizations in LICs, which stabilizes the SEI and prevent counter ions intercalations that leads to graphite exfoliation. [65] Meanwhile, high concentration of hydroxyl and carboxyl functional groups help catalyze the oxygen reduction reactions (ORR) in fuel cells. [66] The differences in the effect brought by different surface oxygen functional groups at the surface of graphite on different electrochemical systems that has been briefly showcased here, along with the trade-off between various properties within 
an electrochemical system highlight the importance of fine tuning the structure and composition of oxygen functional groups on the surface of graphite to obtain optimum performances of electrochemical systems.

\section{Conclusions}

We have investigated the stability of hydroxyl, ketonic, carboxyl, and carbonyl functional groups on both edge and basal sites of a graphite anode as a function of surface coverage. A series of DFT calculations reveal that at typical operating conditions of LIBs, the armchair edge of an uncharged graphite anode will be fully functionalized by oxygen functional groups, while the basal site will remain bare. Surface Pourbaix diagrams show that the oxygen functionals at the edge sites of graphite will be mostly composed of hydroxides and ketones, while carboxyl and carbonyl functional groups will only be present in small amounts. Furthermore, we observe the transformation of carbonyl groups into ketonic groups in the presence of empty surface carbon sites next to the adsorbed carbonyl groups. The transformation of carbonyl into ketonic groups will further stabilize the graphite surface. In contrast, carboxyl groups are more stable when all surface sites within a carboxyl layer are all populated. On the other hand, the adsorption of oxygen functional groups at the basal sites of graphite requires the application of an external potential. Due to its highly unfavorable thermodynamics, the adsorption of oxygen functional groups at the basal sites will only be in the dilute region.

Assessment of the stability of each functional group across different surface coverages shows a correlation between the stability and surface coverage, where, in general, stability of a specific group increases as the surface coverage increases. Our calculations also unveil the sensitivity of the edge sites to the change in the amount of adsorbed hydroxyl and ketonic functional groups. In the cases of carboxyl and carbonyl functional groups, the adsorption would only have a minor effect on the stability of graphite, unless the adsorbed amounts are in the extremes. We also found that the adsorption sites of oxygen functional groups do not affect the stability of edge graphite, while the basal surface prefers to have all the adsorbed groups cluster together in a highly ordered fashion. In the case of hydroxyl groups adsorbed on 
the graphite basal plane, the $-\mathrm{OH}$ constituents will adsorb on the basal surface in a meta- configuration relative to one another, while the -H constituents will occupy the ortho- sites. Although the process is highly unfavorable, for LIB applications it is important for a small concentration of oxygen functional groups to be adsorbed on the basal sites of graphite during the synthesis to provide structural supports to the anode structure and ensure a fast charge rate.

Seeing that different surface oxygen functional groups affect different aspects of an LIB in different ways, it is of utmost importance that the behavior of different oxygen functional groups on a graphite surface is thoroughly understood. Our findings shed light on the importance of fine tuning the composition and concentration of oxygen functional groups at the surface of a graphite anode to obtain greater control of the performance of LIBs. In the future, this information could be used for rational modification of the graphite/electrolyte interface design to achieve a more superior SEI.

\section{Acknowledgements}

This research was entirely supported by the U.S. Department of Energy, Office of Science, Basic Energy Sciences, CPIMS Program, under Award DE-SC0019483. Computational resources for this work were provided by the Hyak supercomputer system of University of Washington and National Energy Research Scientific Computing Center (NERSC), a U.S. Department of Energy Office of Science User Facility operated under Contract No. DE-AC02-05CH11231. The authors would like to thank Dr. Jesse Prelesnik and Dr. Stephanie Hare for helpful discussion and careful reading of this manuscript.

\section{References}

[1] M. Emmel, C.G. Aneziris, Development of novel carbon bonded filter compositions for steel melt filtration, Ceram. Int. 38 (2012) 5165-5173. https://doi.org/10.1016/j.ceramint.2012.03.022.

[2] K. Xu, A. Von Wald Cresce, $\mathrm{Li}+$-solvation/desolvation dictates interphasial processes on graphitic anode in Li ion cells, J. Mater. Res. 27 (2012) 2327-2341. https://doi.org/10.1557/jmr.2012.104.

[3] D.M. Kabtamu, J.Y. Chen, Y.C. Chang, C.H. Wang, Water-activated graphite felt as a highperformance electrode for vanadium redox flow batteries, J. Power Sources. 341 (2017) 270-279. https://doi.org/10.1016/j.jpowsour.2016.12.004.

[4] C.E.L. Foss, A.M. Svensson, S. Sunde, F. Vullum-Bruer, Edge/basal/defect ratios in graphite and their influence on the thermal stability of lithium ion batteries, J. Power Sources. 317 (2016) $177-$ 183. https://doi.org/10.1016/j.jpowsour.2016.03.079.

[5] S. Thinius, M.M. Islam, T. Bredow, Reconstruction of low-index graphite surfaces, Surf. Sci. 649 (2016) 60-65. https://doi.org/10.1016/j.susc.2016.01.027.

[6] J. Yan, B.J. Xia, Y.C. Su, X.Z. Zhou, J. Zhang, X.G. Zhang, Phenomenologically modeling the formation and evolution of the solid electrolyte interface on the graphite electrode for lithium-ion 
batteries, Electrochim. Acta. 53 (2008) 7069-7078.

https://doi.org/10.1016/j.electacta.2008.05.032.

[7] M.J. McAllister, J.L. Li, D.H. Adamson, H.C. Schniepp, A.A. Abdala, J. Liu, M. Herrera-Alonso, D.L. Milius, R. Car, R.K. Prud'homme, I.A. Aksay, Single sheet functionalized graphene by oxidation and thermal expansion of graphite, Chem. Mater. 19 (2007) 4396-4404.

https://doi.org/10.1021/cm0630800.

[8] E. Peled, Improved Graphite Anode for Lithium-Ion Batteries Chemically, J. Electrochem. Soc. 143 (1996) L4-L7. https://doi.org/10.1149/1.1836372.

[9] I. Dékány, T. Szabó, O. Berkesi, P. Forgó, K. Josepovits, Y. Sanakis, D. Petridis, Evolution of surface functional groups in a series of progressively oxidized graphite oxides, Chem. Mater. 18 (2006) 2740-2749. https://doi.org/10.1021/cm060258.

[10] T. Szabó, E. Tombácz, E. Illés, I. Dékány, Enhanced acidity and pH-dependent surface charge characterization of successively oxidized graphite oxides, Carbon. 44 (2006) 537-545. https://doi.org/10.1016/j.carbon.2005.08.005.

[11] T.H. Kim, E.K. Jeon, Y. Ko, B.Y. Jang, B.S. Kim, H.K. Song, Enlarging the d-spacing of graphite and polarizing its surface charge for driving lithium ions fast, J. Mater. Chem. A. 2 (2014) 7600 7605. https://doi.org/10.1039/c3ta15360f.

[12] K. Leung, J.L. Budzien, Ab initio molecular dynamics simulations of the initial stages of solidelectrolyte interphase formation on lithium ion battery graphitic anodes, Phys. Chem. Chem. Phys. 12 (2010) 6583-6586. https://doi.org/10.1039/b925853a.

[13] S.J. An, J. Li, C. Daniel, D. Mohanty, S. Nagpure, D.L. Wood, The state of understanding of the lithium-ion-battery graphite solid electrolyte interphase (SEI) and its relationship to formation cycling, Carbon. 105 (2016) 52-76. https://doi.org/10.1016/j.carbon.2016.04.008.

[14] Z. Wang, B. Yang, Y. Wang, Y. Zhao, X.M. Cao, P. Hu, Identifying the trend of reactivity for sp2 materials: An electron delocalization model from first principles calculations, Phys. Chem. Chem. Phys. 15 (2013) 9498-9502. https://doi.org/10.1039/c3cp51375k.

[15] C. Uthaisar, V. Barone, B.D. Fahlman, On the chemical nature of thermally reduced graphene oxide and its electrochemical Li intake capacity, Carbon. 61 (2013) 558-567. https://doi.org/10.1016/j.carbon.2013.05.037.

[16] K.A. Mkhoyan, A.W. Contryman, J. Silcox, D.A. Stewart, G. Eda, C. Mattevi, S. Miller, M. Chhowalla, Atomic and electronic structure of graphene-oxide, Nano Lett. 9 (2009) 1058-1063. https://doi.org/10.1021/n18034256.

[17] A.Y.S. Eng, C.K. Chua, M. Pumera, Refinements to the structure of graphite oxide: Absolute quantification of functional groups via selective labelling, Nanoscale. 7 (2015) 20256-20266. https://doi.org/10.1039/c5nr05891k.

[18] K.H. Wu, D.W. Wang, I.R. Gentle, Revisiting oxygen reduction reaction on oxidized and unzipped carbon nanotubes, Carbon. 81 (2015) 295-304.

https://doi.org/10.1016/j.carbon.2014.09.060.

[19] D. Heymann, R.B. Weisman, Fullerene oxides and ozonides, Comptes Rendus Chim. 9 (2006) 1107-1116. https://doi.org/10.1016/j.crci.2006.02.003.

[20] X. Gao, J. Jang, S. Nagase, Hydrazine and thermal reduction of graphene oxide: Reaction mechanisms, product structures, and reaction design, J. Phys. Chem. C. 114 (2010) 832-842. https://doi.org/10.1021/jp909284g.

[21] C. Peng, M.P. Mercer, C.K. Skylaris, D. Kramer, Lithium intercalation edge effects and doping implications for graphite anodes, J. Mater. Chem. A. 8 (2020) 7947-7955. https://doi.org/10.1039/c9ta13862e.

[22] N.N. Intan, J. Pfaendtner, Effect of fluoroethylene carbonate additives on the initial formation of the solid electrolyte interphase on an oxygen-functionalized graphitic anode in lithium-ion batteries, ACS Appl. Mater. Interfaces. 13 (2021) 8169-8180.

https://doi.org/10.1021/acsami.0c18414.

[23] C. Uthaisar, D.J. Hicks, V. Barone, Li adsorption on edge-oxidized graphene nanoribbons 
predicted by DFT calculations, Surf. Sci. 619 (2014) 105-113.

https://doi.org/10.1016/j.susc.2013.10.001.

[24] J.K. Nørskov, J. Rossmeisl, A. Logadottir, L. Lindqvist, J.R. Kitchin, T. Bligaard, H. Jónsson, Origin of the overpotential for oxygen reduction at a fuel-cell cathode, J. Phys. Chem. B. 108 (2004) 17886-17892. https://doi.org/10.1021/jp047349j.

[25] J.D. Verink, Simplified procedure for constructing Pourbaix diagrams, in: Uhlig's Corros. Handb. Third Ed., 2011: pp. 93-101. https://doi.org/10.1002/9780470872864.ch7.

[26] K.S. Williams, J.P. Labukas, V. Rodriguez-Santiago, J.W. Andzelm, First principles modeling of water dissociation on $\mathrm{Mg}(0001)$ and development of a $\mathrm{Mg}$ surface Pourbaix diagram, Corrosion. 71 (2015) 209-223. https://doi.org/10.5006/1322.

[27] M. Valiev, E.J. Bylaska, N. Govind, K. Kowalski, T.P. Straatsma, H.J.J. Van Dam, D. Wang, J. Nieplocha, E. Apra, T.L. Windus, W.A. De Jong, NWChem: A comprehensive and scalable opensource solution for large scale molecular simulations, Comput. Phys. Commun. 181 (2010) 14771489. https://doi.org/10.1016/j.cpc.2010.04.018.

[28] J.P. Perdew, K. Burke, M. Ernzerhof, Generalized gradient approximation made simple, Phys. Rev. Lett. 77 (1996) 3865-3868. https://doi.org/10.1103/PhysRevLett.77.3865.

[29] S. Grimme, S. Ehrlich, L. Goerigk, Effect of the damping function in dispersion corrected density functional theory, J. Comput. Chem. 32 (2011) 1456-1465. https://doi.org/10.1002/jcc.21759.

[30] D.R. Hamann, M. Schlüter, C. Chiang, Norm-conserving pseudopotentials, Phys. Rev. Lett. 43 (1979) 1494-1497. https://doi.org/10.1103/PhysRevLett.43.1494.

[31] D.R. Hamann, Generalized norm-conserving pseudopotentials, Phys. Rev. B. 40 (1989) 29802987. https://doi.org/10.1103/PhysRevB.40.2980.

[32] H.J. Monkhorst, J.D. Pack, Special points for Brillouin-zone integrations, Phys. Rev. B. 13 (1976) 5188-5192. https://doi.org/10.1103/PhysRevB.13.5188.

[33] M.C. Payne, M.P. Teter, D.C. Allan, T.A. Arias, J.D. Joannopoulos, Iterative minimization techniques for ab initio total-energy calculations: Molecular dynamics and conjugate gradients, Rev. Mod. Phys. 64 (1992) 1045-1097. https://doi.org/10.1103/RevModPhys.64.1045.

[34] V. Viswanathan, H.A. Hansen, J. Rossmeisl, J.K. Nørskov, Universality in oxygen reduction electrocatalysis on metal surfaces, ACS Catal. 2 (2012) 1654-1660. https://doi.org/10.1021/cs300227s.

[35] K. Leung, Two-electron reduction of ethylene carbonate: A quantum chemistry re-examination of mechanisms, Chem. Phys. Lett. 568 (2013) 1-8. https://doi.org/10.1016/j.cplett.2012.08.022.

[36] M. Nie, J. Demeaux, B.T. Young, D.R. Heskett, Y. Chen, A. Bose, J.C. Woicik, B.L. Lucht, Effect of vinylene carbonate and fluoroethylene carbonate on SEI formation on graphitic anodes in LiIon batteries, J. Electrochem. Soc. 162 (2015) A7008-A7014. https://doi.org/10.1149/2.0021513jes.

[37] B. Zhang, M. Metzger, S. Solchenbach, M. Payne, S. Meini, H.A. Gasteiger, A. Garsuch, B.L. Lucht, Role of 1,3-propane sultone and vinylene carbonate in solid electrolyte interface formation and gas generation, J. Phys. Chem. C. 119 (2015) 11337-11348. https://doi.org/10.1021/acs.jpcc.5b00072.

[38] I. Nayyar, B. Ginovska, A. Karkamkar, T. Gennett, T. Autrey, Physi-sorption of H2 on pure and boron-doped graphene monolayers: a dispersion-corrected DFT study, C - J. Carbon Res. 6 (2020) 15. https://doi.org/10.3390/c6010015.

[39] G. Shi, J. Liu, C. Wang, B. Song, Y. Tu, J. Hu, H. Fang, Ion enrichment on the hydrophobic carbon-based surface in aqueous salt solutions due to cation- $\pi$ interactions, Sci. Rep. 3 (2013) 1-5. https://doi.org/10.1038/srep03436.

[40] J. Rossmeisl, J.K. Nørskov, C.D. Taylor, M.J. Janik, M. Neurock, Calculated phase diagrams for the electrochemical oxidation and reduction of water over Pt(111), J. Phys. Chem. B. 110 (2006) 21833-21839. https://doi.org/10.1021/jp0631735.

[41] H.A. Hansen, J. Rossmeisl, J.K. Nørskov, Surface Pourbaix diagrams and oxygen reduction activity of Pt, Ag and Ni(111) surfaces studied by DFT, Phys. Chem. Chem. Phys. 10 (2008) 
3722-3730. https://doi.org/10.1039/b803956a.

[42] D.M. Chen, P.M. Shenai, Y. Zhao, Tight binding description on the band gap opening of pyrenedispersed graphene, Phys. Chem. Chem. Phys. 13 (2011) 1515-1520.

https://doi.org/10.1039/c0cp00909a.

[43] Y. Xu, B. Tian, S. Fang, W. Guo, Z. Zhang, Probing the interaction of water molecules with oxidized graphene by first principles, J. Phys. Chem. C. 125 (2021) 4580-4587. https://doi.org/10.1021/acs.jpcc.0c10881.

[44] S. Back, S. Siahrostami, Noble metal supported hexagonal boron nitride for the oxygen reduction reaction: A DFT study, Nanoscale Adv. 1 (2019) 132-139. https://doi.org/10.1039/c8na00059j.

[45] G. Gao, A.P. O'Mullane, A. Du, 2D MXenes: A new family of promising catalysts for the hydrogen evolution reaction, ACS Catal. 7 (2017) 494-500. https://doi.org/10.1021/acscatal.6b02754.

[46] I.C. Man, H.Y. Su, F. Calle-Vallejo, H.A. Hansen, J.I. Martínez, N.G. Inoglu, J. Kitchin, T.F. Jaramillo, J.K. Nørskov, J. Rossmeisl, Universality in oxygen evolution electrocatalysis on oxide surfaces, ChemCatChem. 3 (2011) 1159-1165. https://doi.org/10.1002/cctc.201000397.

[47] A. Kulkarni, S. Siahrostami, A. Patel, J.K. Nørskov, Understanding catalytic activity trends in the oxygen reduction reaction, Chem. Rev. 118 (2018) 2302-2312. https://doi.org/10.1021/acs.chemrev.7b00488.

[48] Z. Duan, G. Henkelman, Theoretical resolution of the exceptional oxygen reduction activity of $\mathrm{Au}(100)$ in alkaline media, ACS Catal. 9 (2019) 5567-5573. https://doi.org/10.1021/acscatal.9b00955.

[49] W. Bauer, F.A. Çetinel, M. Müller, U. Kaufmann, Effects of $\mathrm{pH}$ control by acid addition at the aqueous processing of cathodes for lithium ion batteries, Electrochim. Acta. 317 (2019) 112-119. https://doi.org/10.1016/j.electacta.2019.05.141.

[50] Y. Li, Y. Lu, P. Adelhelm, M.M. Titirici, Y.S. Hu, Intercalation chemistry of graphite: Alkali metal ions and beyond, Chem. Soc. Rev. 48 (2019) 4655-4687. https://doi.org/10.1039/c9cs00162j.

[51] F.A. Do Amaral, É.O. Machado, L.L. De Freitas, L.K. Santana, S.C. Canobre, Synthesis of Co-Al$\mathrm{Cl}$ LDH by cathodic material reprocessing from cellular phone batteries, Mater. Res. 17 (2014) 914. https://doi.org/10.1590/S1516-14392014005000049.

[52] H.R. Jiang, M.C. Wu, Y.X. Ren, W. Shyy, T.S. Zhao, Towards a uniform distribution of zinc in the negative electrode for zinc bromine flow batteries, Appl. Energy. 213 (2018) 366-374. https://doi.org/10.1016/j.apenergy.2018.01.061.

[53] H.C. Schniepp, J.L. Li, M.J. McAllister, H. Sai, M. Herrera-Alonson, D.H. Adamson, R.K. Prud'homme, R. Car, D.A. Seville, I.A. Aksay, Functionalized single graphene sheets derived from splitting graphite oxide, J. Phys. Chem. B. 110 (2006) 8535-8539. https://doi.org/10.1021/jp060936f.

[54] S.J. An, J. Li, C. Daniel, D.L. Wood, Effects of ultraviolet light treatment in ambient air on Lithium-Ion battery graphite and PVDF binder, J. Electrochem. Soc. 166 (2019) A1121-A1126. https://doi.org/10.1149/2.0591906jes.

[55] E. Ventosa, W. Xia, S. Klink, F. La Mantia, M. Muhler, W. Schuhmann, Influence of surface functional groups on lithium ion intercalation of carbon cloth, Electrochim. Acta. 65 (2012) $22-$ 29. https://doi.org/10.1016/j.electacta.2011.12.128.

[56] S. Rustam, N.N. Intan, J. Pfaendtner, Effect of graphitic anode surface functionalization on the structure and dynamics of electrolytes at the interface, J. Chem. Phys. (2021) under review.

[57] Q. Wang, B. Mao, S.I. Stoliarov, J. Sun, A review of lithium ion battery failure mechanisms and fire prevention strategies, Prog. Energy Combust. Sci. 73 (2019) 95-131. https://doi.org/10.1016/j.pecs.2019.03.002.

[58] Z. Xie, Z. Yu, W. Fan, G. Peng, M. Qu, Effects of functional groups of graphene oxide on the electrochemical performance of lithium-ion batteries, RSC Adv. 5 (2015) 90041-90048. https://doi.org/10.1039/c5ra17854a. 
[59] C.E.L. Foss, S. Müssig, A.M. Svensson, P.J.S. Vie, A. Ulvestad, J.P. Mæhlen, A.Y. Koposov, Anodes for Li-ion batteries prepared from microcrystalline silicon and enabled by binder's chemistry and pseudo-self-healing, Sci. Rep. 10 (2020) 1-8. https://doi.org/10.1038/s41598-02070001-5.

[60] B. Gendensuren, E.S. Oh, Dual-crosslinked network binder of alginate with polyacrylamide for silicon/graphite anodes of lithium ion battery, J. Power Sources. 384 (2018) 379-386. https://doi.org/10.1016/j.jpowsour.2018.03.009.

[61] M. Ling, J. Qiu, S. Li, H. Zhao, G. Liu, S. Zhang, An environmentally benign LIB fabrication process using a low cost, water soluble and efficient binder, J. Mater. Chem. A. 1 (2013) 1154311547. https://doi.org/10.1039/c3ta12159c.

[62] Y. Matsuo, J. Taninaka, K. Hashiguchi, T. Sasaki, Q. Cheng, Y. Okamoto, N. Tamura, Effect of oxygen contents in graphene like graphite anodes on their capacity for lithium ion battery, $\mathrm{J}$. Power Sources. 396 (2018) 134-140. https://doi.org/10.1016/j.jpowsour.2018.06.022.

[63] H.S. Moon, J.H. Lee, S. Kwon, I.T. Kim, S.G. Lee, Mechanisms of Na adsorption on graphene and graphene oxide: Density functional theory approach, Carbon Lett. 16 (2015) 116-120. https://doi.org/10.5714/CL.2015.16.2.116.

[64] A.S. Dobrota, I.A. Pašti, N. V. Skorodumova, Oxidized graphene as an electrode material for rechargeable metal-ion batteries - a DFT point of view, Electrochim. Acta. 176 (2015) 1092-1099. https://doi.org/10.1016/j.electacta.2015.07.125.

[65] J.H. Lee, W.H. Shin, S.Y. Lim, B.G. Kim, J.W. Choi, Modified graphite and graphene electrodes for high-performance lithium ion hybrid capacitors, Mater. Renew. Sustain. Energy. 3 (2014) 1-8. https://doi.org/10.1007/s40243-014-0022-9.

[66] A.B. Soliman, H.S. Abdel-Samad, S.S.A. Rehim, H.H. Hassan, Surface functionality and electrochemical investigations of a graphitic electrode as a candidate for alkaline energy conversion and storage devices, Sci. Rep. 6 (2016) 1-12. https://doi.org/10.1038/srep22056. 


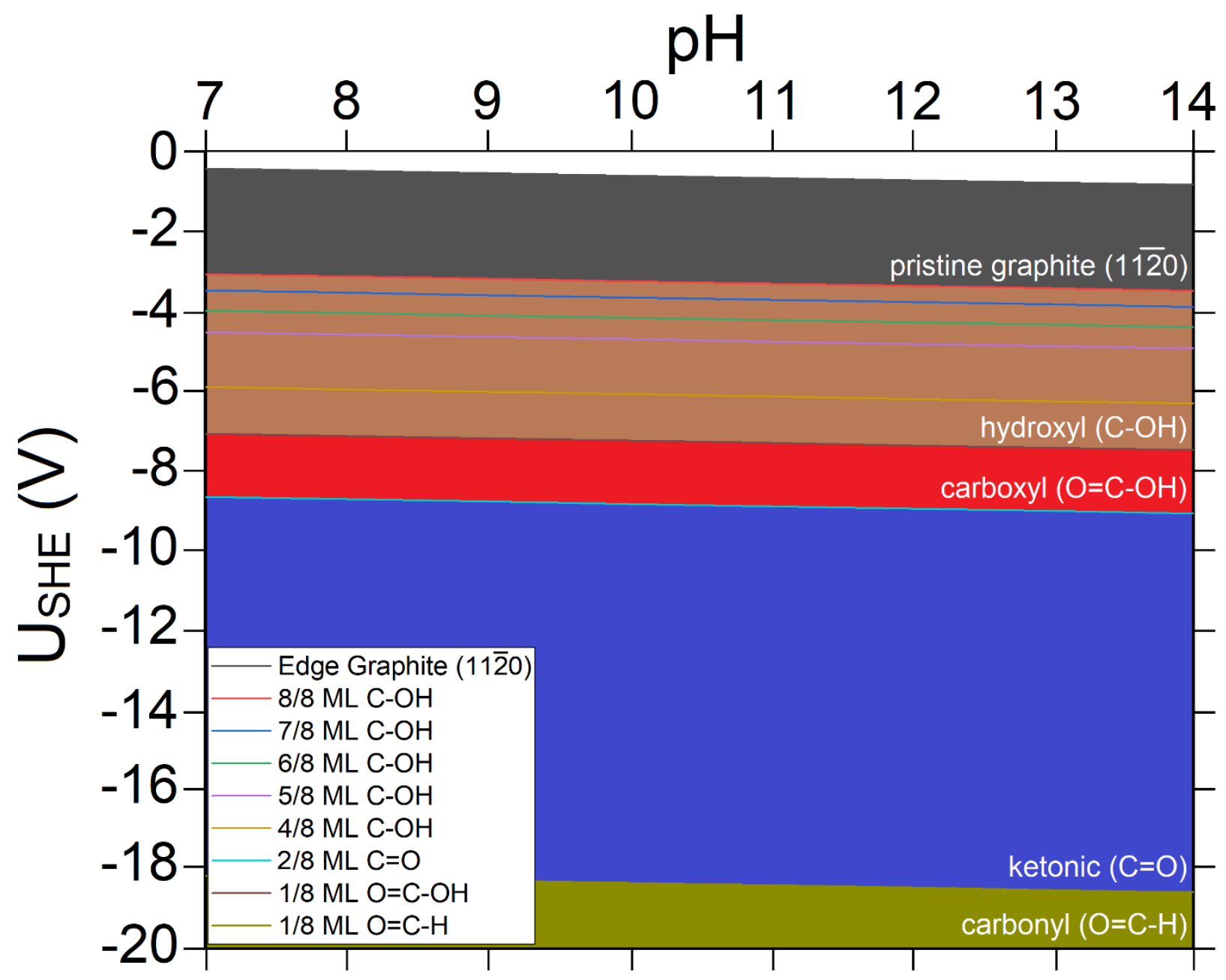

$\underline{\text { Graphical Abstract }}$ 